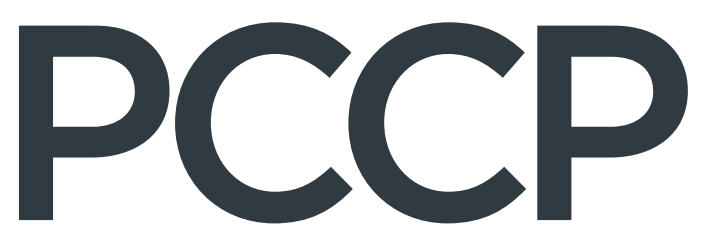

Physical Chemistry Chemical Physics

rsc.li/pccp

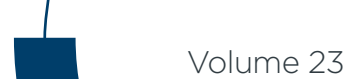

Number 26

14 July 2021

Pages 14113-14534

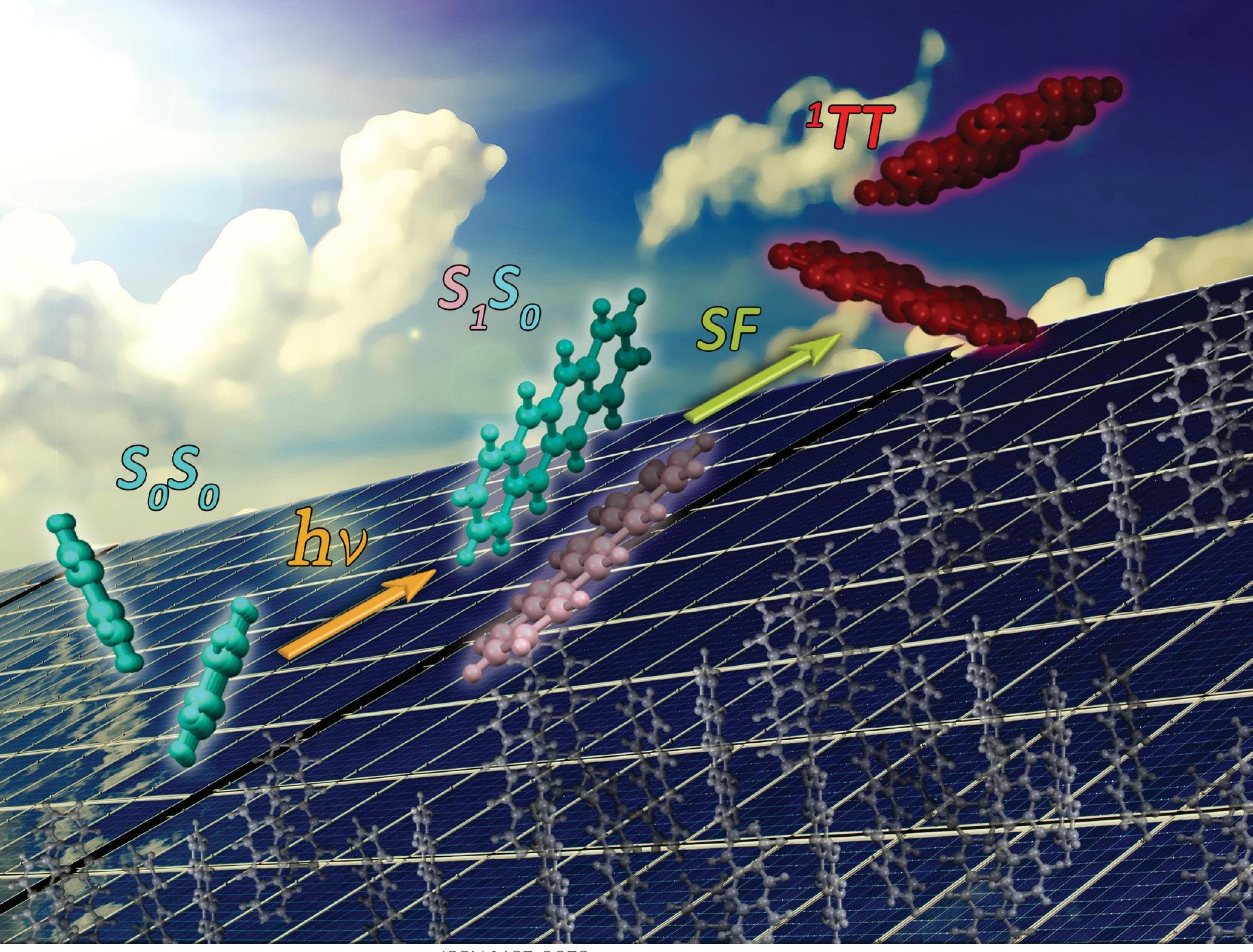

ISSN 1463-9076

\title{
PAPER
}

Shirin Faraji et al.

Influence of the crystal packing in singlet fission: one step

beyond the gas phase approximation 
Check for updates

Cite this: Phys. Chem. Chem. Phys., 2021, 23, 14164

Received 21st January 2021 Accepted 9th May 2021

DOI: $10.1039 / \mathrm{d} 1 \mathrm{cp} 00298 \mathrm{~h}$

rsc.li/pccp

\section{Influence of the crystal packing in singlet fission: one step beyond the gas phase approximation $\uparrow$}

\author{
Luis Enrique Aguilar Suarez, (DD ${ }^{a}$ Coen de Graaf (D) abc and Shirin Faraji (DD *a
}

\section{Introduction}

Conversion of sunlight into electricity remains as one of the most promising sources to generate renewable and clean energy. ${ }^{1,2}$ In fact, it has been estimated that if we were able to harvest the amount of energy that the sun delivers during one hour to the Earth's surface, we would fulfil the world's total annual energy consumption. ${ }^{3}$ Nevertheless, the artificial sunlightharvesting is still problematic due to the low conversion yields shown by current solar cells where challenges related to thermalisation loss, exciton diffusion and dissociation and charge collection need to be addressed. ${ }^{4}$ Therefore, ways to improve their efficiency are needed. Among all the proposed solutions, singlet fission (SF) has been considered as a potential scheme to overcome the so-called Shockley-Queisser limit of $34 \%$ on the efficiency of single-junction solar cells, since two pairs of charge carriers can be generated per single absorbed photon. ${ }^{5-8}$ Theoretical estimations suggest that the conversion yield of a single-junction photovoltaic device could

\footnotetext{
${ }^{a}$ Theoretical Chemistry Group, Zernike Institute for Advanced Materials, University of Groningen, Nijenborgh 4, 9747 AG Groningen, The Netherlands. E-mail: s.s.faraji@rug.nl

${ }^{b}$ Department of Physical and Inorganic Chemistry, Universitat Rovira $i$ Virgili, Campus Sescelades, C. Marcel li Domingo 1, 43007 Tarragona, Spain

${ }^{c}$ ICREA, Pg. Lluis Companys 23, 08010 Barcelona, Spain

$\dagger$ Electronic supplementary information (ESI) available: NOCI and AIFDEM coefficients and Cartesian coordinates for the relevant dimers and trimers. See DOI: $10.1039 / \mathrm{d} 1 \mathrm{cp} 00298 \mathrm{~h}$
}

increase up to $45 \%$ after inclusion of a SF material layer. ${ }^{9}$ If endothermic SF is also considered in the estimation, the efficiency has been calculated to increase to $45.9 \% .{ }^{10}$ In fact, experiment has already shown the potential that adding SF materials has on increasing the efficiency in different photovoltaic architectures. ${ }^{11,12}$

SF takes place typically in organic solids and a general scheme is depicted in Fig. 1. In this process, a singlet excited $\left(S_{1}\right)$ chromophore, generated upon absorption of a single photon by the molecule in its ground state $\left(\mathrm{S}_{0}\right)$, transfers part of its energy to a neighbour chromophore to form two coupled triplets (the so-called ${ }^{1}$ TT state) in an overall spin-allowed process. ${ }^{5,6}$ The conversion efficiency to the ${ }^{1}$ TT state depends mainly on two factors; First, the SF process should be preferably slightly exothermic (although endothermic SF has also been investigated ${ }^{13,14}$ ) which means that the energy condition $\Delta E\left(\mathrm{~S}_{1}\right)>2 \Delta E\left(\mathrm{~T}_{1}\right)$ has to be fulfilled for the individual chromophore. Second, the mutual disposition of the chromophores plays an essential role in both the energy levels and the electronic coupling between the relevant diabatic states. Theoretical efforts, at different levels of sophistication, have been made to find novel chromophores that fulfil the SF energy condition $^{15-21}$ as well as protocols to optimise the spatial distribution of the chromophores and maximise the coupling between $\mathrm{S}_{0} \mathrm{~S}_{1}$ (or $\mathrm{S}_{1} \mathrm{~S}_{0}$ ) and ${ }^{1} \mathrm{TT}^{22-25}$

The simple picture in Fig. 1 is generally accepted, but the conversion from $\mathrm{S}_{0} \mathrm{~S}_{1}$ (or $\mathrm{S}_{1} \mathrm{~S}_{0}$ ) to the ${ }^{1} \mathrm{TT}$ state is still subject of ongoing debate. Three different mechanisms have been proposed to explain the process: ${ }^{26-30}$ (i) the direct mechanism 


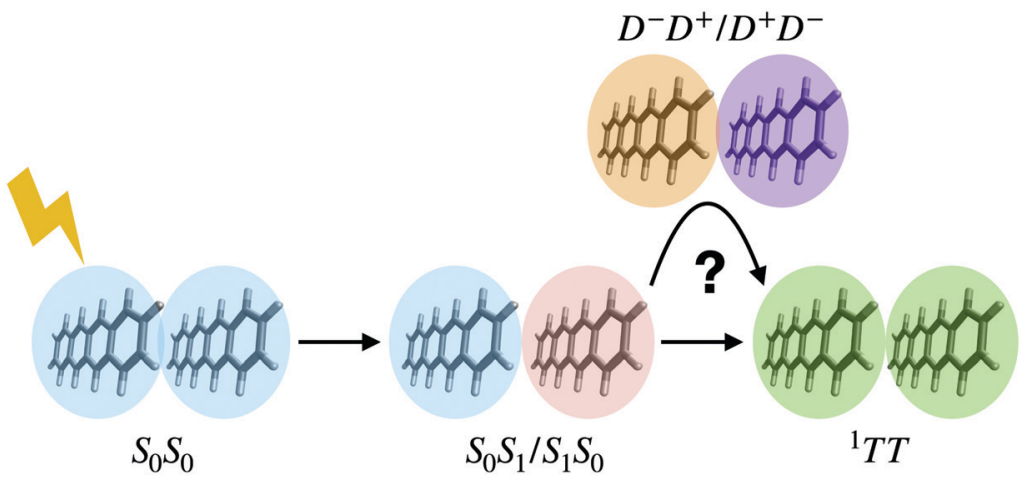

Fig. 1 Singlet fission process: upon absorption of a photon, one photoexcited $\left(\mathrm{S}_{1}\right)$ chromophore transfers part of its energy to a neighbour in the ground state $\left(S_{0}\right)$ to form two coupled triplets $\left({ }^{1} T T\right)$ in a spin-allowed process. Charge transfer states $\left(D^{-} D^{+}\right.$and $\left.D^{+} D^{-}\right)$are believed to play an essential role.

in which there is straight conversion via a two-electron transfer; (ii) the mediated mechanism, in which high-energy charge transfer $(\mathrm{CT})$ states $\left(\mathrm{D}^{+} \mathrm{D}^{-}\right.$and $\mathrm{D}^{-} \mathrm{D}^{+}$in Fig. 1) are believed to mix with the initial and final states enhancing their coupling through the so-called superexchange effect; and finally (iii) a two-step mechanism known as charge hopping in which CT states act as intermediates and are populated before the generation of the ${ }^{1}$ TT state. Thus, understanding how the coupled triplets are generated is crucial to design guidelines for optimising the conditions for the formation of the ${ }^{1} \mathrm{TT}$ state.

Several theoretical and computational studies have been performed to understand the underlying mechanism of SF. These works focus either on the simple model of two interacting chromophores in the gas phase $e^{23,27,31-35}$ or by studying the process taking into account the crystal packing applying a variety of methods. ${ }^{36-39}$ The gas phase dimer model has been proven to be useful as a first approximation to study SF. However, its reliability for the description of the process in organic crystals needs further analysis and validation.

An early study on the acenes family employing a simple kinetic model ${ }^{32}$ has pointed out that the SF yield depends on different factors, such as couplings, singlet-triplet energy gaps and entropic factors with the latter being an important contribution to be considered. A previous study on pentacene $\mathrm{J}$-type and H-type ring-shaped aggregates have concluded that the electronic coupling depends heavily on both the size and structure of the aggregate. ${ }^{40}$ These findings show the importance of going beyond the dimer model. Furthermore, the influence of the environment has been studied previously in model crystals for tetracene and pentacene. The findings suggest that $\mathrm{SF}$ can occur without the presence of a low-lying CT state in the acene crystal and that for tetracene the ${ }^{1} \mathrm{TT}$ state is higher in energy than $\mathrm{S}_{1}$ and $\mathrm{S}_{2}{ }^{41}$ Nevertheless, a recent work has pointed to the presence of low-lying CT states in pentacene clusters that are energetically available when increasing the cluster size and that depend on the choice of functional. ${ }^{42}$ These conclusions encourage to study the SF process taking into account the crystal environment.

Here, we present a study of the influence of crystal packing on the electronic couplings, the so-called exciton descriptors and the overall underlying SF mechanism on pairs of tetracene $e^{43-47}$ and 1,3-diphenylisobenzofuran (DPBF),$^{35,48-50}$ two chromophores known to exhibit SF. This contribution is organized as follows: the approach employed to evaluate the electronic couplings for the pairs of molecules is presented in Section 1.1. In Section 1.2, a subset of the so-called exciton descriptors and how to apply them to assign the character of excited states is described. The approaches used for the inclusion of the environment are mentioned in each of these sections. Results of the influence of the environment on the electronic couplings and exciton descriptors for pairs of the two different chromophores are discussed in Section 3. Concluding remarks of this contribution are given in Section 4.

\subsection{Non-orthogonal configuration interaction approach}

A SF rate $\left(k_{\mathrm{E}}\right)$ in a system can be approximated by means of Fermi's golden rule: ${ }^{51}$

$$
k_{\mathrm{E}}=\frac{2 \pi}{\hbar}|V|^{2} \rho(E)
$$

where $V$ denotes the electronic coupling between the relevant diabatic states and $\rho(E)$ represents the density of states per energy $(E)$ unit. Different approaches have been used to evaluate the electronic coupling, ${ }^{5,6,22,52-54}$ but in this work a non-orthogonal configuration interaction (NOCI) approach is employed. ${ }^{55,56}$ The advantages of using this method include a clear chemical interpretation of the states and the compactness of wave functions since each of the molecular states are described with its own optimal set of orbitals. ${ }^{57}$

The NOCI wave function describing the molecular pair can be expanded as a linear combination of the so-called manyelectron basis functions (MEBFs, $\Phi$ ) which describe different electronic states of the pair. These MEBFs are generated as antisymmetrised products of molecular wave functions:

$$
\Phi_{\mu}=\hat{A}\left|\Psi_{\alpha \mathrm{A}} \Psi_{\beta \mathrm{B}}\right|
$$

where $\alpha$ and $\beta$ represent the electronic states of molecules $\mathrm{A}$ and $\mathrm{B}$, respectively. In this contribution, the molecular wave functions are chosen to be of the complete active space selfconsistent field (CASSCF) type. For the SF process, the following six electronic states are of particular interest: $\Phi_{\mathrm{S}_{0} \mathrm{~S}_{0}}, \Phi_{\mathrm{S}_{0} \mathrm{~S}_{1}}, \Phi_{\mathrm{S}_{1} \mathrm{~S}_{0}}$, $\Phi_{1 \mathrm{TT}}, \Phi_{\mathrm{D}^{+} \mathrm{D}^{-}}$and $\Phi_{\mathrm{D}^{-} \mathrm{D}^{+}}$. Subsequently, Hamiltonian and overlap 
matrix elements between the MEBFs are calculated. The electronic coupling can be then computed by the means of the direct coupling scheme: ${ }^{58}$

$$
V=\frac{\left\langle\Phi_{\mu}|\hat{H}| \Phi_{v}\right\rangle-\frac{1}{2}\left(\left\langle\Phi_{\mu}|\hat{H}| \Phi_{\mu}\right\rangle+\left\langle\Phi_{v}|\hat{H}| \Phi_{v}\right\rangle\right) \cdot\left\langle\Phi_{\mu} \mid \Phi_{v}\right\rangle}{1-\left\langle\Phi_{\mu} \mid \Phi_{v}\right\rangle^{2}}
$$

where $\mu$ and the $\nu$ represent the local excited states and the ${ }^{1} \mathrm{TT}$ state, respectively. We use the embedded cluster approach ${ }^{59,60}$ to account for the surrounding crystal environment. The orbital optimisation and generation of the molecular wave functions of the electronic states of the molecules are performed using a frozen Hartree-Fock (HF) electron density around the target molecule.

\subsection{Exciton descriptors}

In addition to the excited state of local singlet coupled triplets, there are four more states that can play a role in the basic description of the singlet fission process. The character of these states is depicted in Fig. 2. ${ }^{61}$ Two of them correspond to local excitons on each of the chromophores $\left(\mathrm{S}_{0} \mathrm{~S}_{1}\right.$ and $\left.\mathrm{S}_{1} \mathrm{~S}_{0}\right)$ and the other two are separated CT states $\left(\mathrm{D}^{+} \mathrm{D}^{-}\right.$and $\left.\mathrm{D}^{-} \mathrm{D}^{+}\right)$. Linear combinations of these states can be constructed under resonance conditions, leading to excitonic resonance (ER) and charge resonance (CR) states. ${ }^{61}$ Please note that the presence of LE states in the dimer excludes the ER states (and vice versa), and similar for the CT and CR states that are also mutually exclusive. An excited state analysis can be done in order to identify and differentiate between the state character.

Recently developed excited state analysis based on the oneparticle transition density matrix (1TDM) offers a compact description of the character of electronic transitions. ${ }^{62,63}$ This analysis has the following advantages: ${ }^{64}$ (1) it can be applied to any excited state method that provides access to the 1TDM, (2) allows a detailed benchmarking of excited state methods, and (3) facilitates the characterisation of excited states of a molecule or a cluster of molecules. The character assignment can be done based on the so-called exciton descriptors. ${ }^{61,65-69}$ In a previous work, we have compared a subset of these descriptors calculated with time-dependent density functional theory (TD-DFT) against wave function-based methods for the tetracene molecule and pairs, establishing CAM-B3LYP/ANO-S-VDZP as the

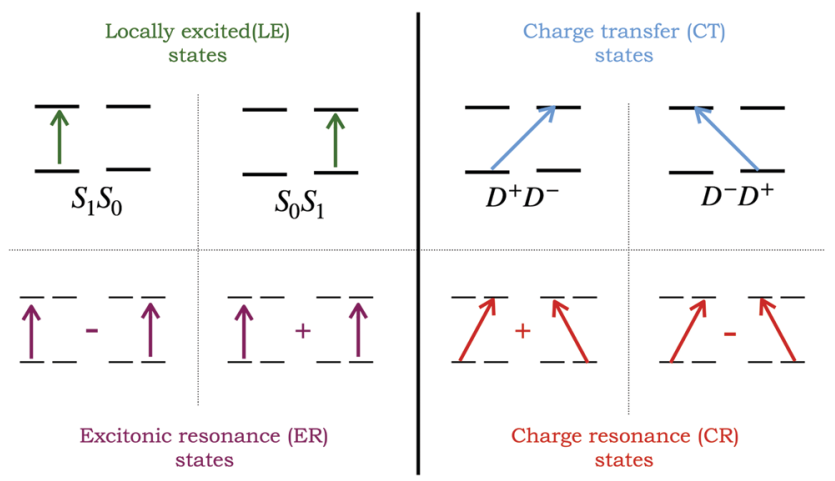

Fig. 2 Localised excited states and their linear combinations leading to delocalised states in the pair model.

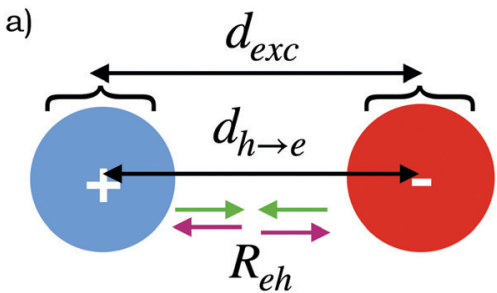

b)

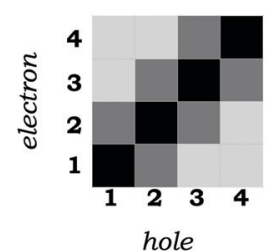

Fig. 3 (a) Depiction of the subset of exciton descriptors employed in this contribution, and (b) general representation of the electron-hole correlation plots with a fragmentation scheme of $n=4$ as an example.

most suitable theoretical method. ${ }^{70}$ Here, we will focus on describing the exciton descriptors depicted in panel (a) of Fig. 3 and how these can be used to assign the character of excited states. For a more elaborate discussion on excited-state descriptors, the reader is referred to the ref. 62-64.

A descriptor related to the amount of CT character in an excited state is the vectorial distance $\left(d_{\mathrm{h} \rightarrow \mathrm{e}}\right)$ between the centroids of the hole and electron. This quantity is particularly useful to identify CT states: $d_{\mathrm{h} \rightarrow \mathrm{e}} \approx 0$ indicates a locally excited (LE) state whereas $d_{\mathrm{h} \rightarrow \mathrm{e}}>0$ indicates a completely separated CT state. The magnitude of $d_{\mathrm{h} \rightarrow \mathrm{e}}$ (in $\AA$ ) can be often associated with the distance of separation between the fragments where the CT takes place, e.g. it can be close to the distance of separation between the chromophores if the CT occurs in a pair of molecules.

Detection of CR states can be challenging but the exciton size $\left(d_{\text {exc }}\right)$ has been proven to be helpful in their characterisation. ${ }^{61}$ This descriptor is the root-mean-square distance between the hole and electron, and takes into account not only the separation between the centroids of hole and electron but also their spatial distribution as well as their covariance. ${ }^{64}$ It is advisable to look at this descriptor to identify hidden resonance states, e.g. $d_{\mathrm{exc}}$ values for ER and CR states are rather different and easily spotted by comparison.

$R_{\mathrm{eh}}$ is known as the correlation coefficient and its value ranges from -1 to 1 . Its sign provides information about the exciton correlation. Positive values indicate that the hole and electron are attracted to each other by Coulombic forces and that they move together as an entity. On the contrary, negative values suggest that the electron and hole avoid each other in space arising from exchange repulsion or correlation effects. ${ }^{69}$ If its value is zero, there is no correlation between the hole and the electron, and they move independently from each other.

Electron-hole correlation plots are pseudo-matrix representations of the so-called charge transfer numbers $\left(\Omega_{\mathrm{AB}}\right) . \Omega_{\mathrm{AB}}$ are calculated between a priori defined fragments in which the molecule or system is split. Panel (b) of Fig. 3 shows a $4 \times 4$ electron-hole correlation plot of a system split into four fragments. Local excitations correspond to the diagonal elements of the matrix going from the bottom-left to upper-right, whereas off-diagonal elements represent CT occurring between the corresponding fragments. In this contribution, the intensity of the transition between and within the fragments is marked in a grey scale. Another useful descriptor results from summing up the off-diagonal $\Omega_{\mathrm{AB}}$ values to obtain the so-called total 
charge transfer number $\left(\omega_{\mathrm{CT}}\right)$. Its values go from zero to one, with $\omega_{\mathrm{CT}}=0$ indicating a pure LE state and $\omega_{\mathrm{CT}}=1$ a state with CT character.

For the inclusion of the crystal environment in the calculation of the exciton descriptors, we employ a hybrid quantum mechanics/ molecular mechanics (QM/MM) approach with the electrostatic embedding scheme. ${ }^{71}$ The pairs are included in the QM subsystem whereas the surrounding crystal environment is treated as point charges in the MM subsystem.

\section{Computational details}

\section{Calculation of electronic couplings}

For the gas phase calculations, CASSCF(6,6)/ANO-S-VDZP molecular wave functions for the three tetracene molecules (highlighted in Fig. 5) describing the ground state $\left(S_{0}\right)$, the first excited state $\left(\mathrm{S}_{1}\right)$, the lowest triplet state $\left(\mathrm{T}_{1}\right)$, the cationic $\left(\mathrm{D}^{+}\right)$and anionic $\left(\mathrm{D}^{-}\right)$forms were generated with the OpenMolcas ${ }^{72,73}$ package. The geometries of the three tetracene molecules were taken directly from the crystal structure (CCDC code TETCEN). Please note that these geometries were used for the calculations of all the molecular states. In order to reduce the computational cost associated with the NOCI calculations, we have followed the protocol as explained in ref. 74 to generate a reduced common molecular orbital (MO) basis for each of the three possible pairs. The common basis was generated with a program written in f95, and the molecular wave functions of the electronic states are now expressed in the new MO basis. For the three pairs, construction of the following six MEBFs: $\Phi_{\mathrm{S} 0 \mathrm{~S} 0}, \Phi_{\mathrm{SoS} 1}, \Phi_{\mathrm{S} 1 \mathrm{~S} 0}, \Phi_{1 \mathrm{TT}}, \Phi_{\mathrm{D}^{+} \mathrm{D}^{-}}$and $\Phi_{\mathrm{D}^{-} \mathrm{D}^{+}}$, and calculation of the Hamiltonian and overlap matrix elements are performed with the GronOR ${ }^{55,56}$ code. Finally, the electronic couplings are calculated as defined in eqn (3) between the localised excited states and the ${ }^{1}$ TT state. Two diabatic states, $\Phi_{\mathrm{S}[1]}$ and $\Phi_{\mathrm{S}[2]}$, were constructed from a $2 \times 2$ NOCI calculation in the basis of the $\Phi_{\mathrm{S} 0 \mathrm{~S} 1}$ and $\Phi_{\mathrm{S} 1 \mathrm{~S} 0}$ MEBFs, whereas the diabatic state of the coupled triplets corresponds to the $\Phi_{1_{\mathrm{TT}}}$ MEBF. Note that the $\mathrm{S}[1]$ and $\mathrm{S}[2]$ diabats are not necessarily the simple +/combinations of the $\mathrm{S}_{0} \mathrm{~S}_{1}$ and $\mathrm{S}_{1} \mathrm{~S}_{0}$ MEBFs as there is no inversion center between the pair of molecules. The importance of the charge transfer states in the description of the lower lying $\mathrm{S}[1]$, $\mathrm{S}[2]$ and ${ }^{1}$ TT states is explored by performing a $4 \times 4$ NOCI calculation with the $\Phi_{\mathrm{S} 0 \mathrm{~S} 1}, \Phi_{\mathrm{S} 1 \mathrm{~S} 0}$ and $\Phi_{\mathrm{D}^{+} \mathrm{D}^{-}}, \Phi_{\mathrm{D}^{+} \mathrm{D}^{-}}$MEBFs on one hand, and a $3 \times 3$ NOCI with the $\Phi_{1 \mathrm{TT}}$ and $\Phi_{\mathrm{D}^{+} \mathrm{D}^{-}}, \Phi_{\mathrm{D}^{+} \mathrm{D}^{-}}$MEBFs on the other. For the embedded cluster calculations, the frozen HF density for each molecule was generated considering twelve surrounding tetracene molecules with the ANO-S-MB basis set.

\section{Excited state analysis}

Excited state calculations were performed on the three tetracene pairs in the gas phase using TD-DFT within the TammDancoff approximation at the CAM-B3LYP/ANO-S-VDZP level of theory. The excited state calculations were carried out as implemented in the Q-Chem $5.3^{75}$ quantum chemistry package. For the QM/MM calculations, each tetracene pair was included in the QM subsystem and treated at the same level of theory as

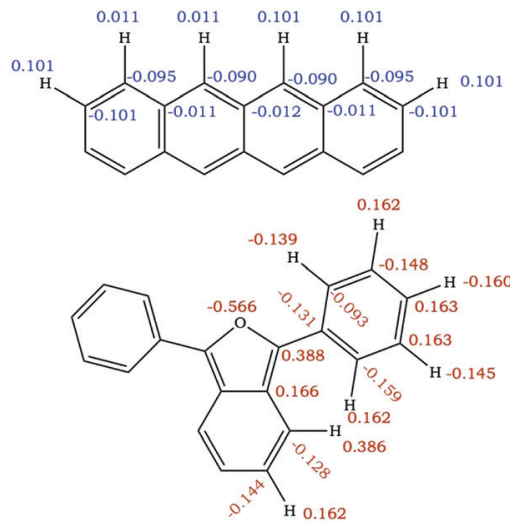

Fig. 4 Point charge values used for the $Q M / M M$ calculations for tetracene (top) and DPBF (bottom). Due to symmetry, only equivalent charges are shown.

in the gas phase. The MM region considered 123 molecules in a symmetrical portion of the crystal. The surrounding molecules were treated as Charge Model 5 point charges $^{76}$ as shown in Fig. 4. Post-processing of results and generation of the electronhole correlation plots were performed with the TheoDORE $2.2^{77,78}$ analysis package. In this contribution, we use a gray scale ranging from black (highest $\Omega_{\mathrm{AB}}$ value) to white (lowest $\Omega_{\mathrm{AB}}$ ) color. For the fragmentation scheme, we considered each molecule as an individual fragment which resulted in $2 \times 2$ electron-hole correlation plots in the case of dimers and in $3 \times 3$ plots for the trimers. For the QM/MM calculations of DPBF, six different QM regions were considered as explained in the next section. For the dimers the MM region included 178 molecules and 177 for the trimers. All relevant Cartesian coordinates are given in the ESI. $\dagger$

\section{Results and discussion}

\section{Tetracene}

Fig. 5 highlights the three pairs considered for the QM calculations $(\mathrm{AB}, \mathrm{AC}$ and $\mathrm{BC})$ within the crystal structure.

Charge transfer mediated electronic couplings calculated for the three tetracene pairs, with and without considering the environment, are reported in Table 1. In the AC and BC pairs, the inclusion of the environment enhances $V$ of the $S[1]$ state by 13 and $17 \mathrm{meV}$, respectively, and approximately $2 \mathrm{meV}$ for $\mathrm{S}[2]$ compared to the gas-phase values. The couplings are within the range of previously reported NOCI values for tetracene. ${ }^{16,57,70}$ The parallel $\mathrm{AB}$ disposition shows the lower $V$ value which translates to the lowest probability of SF to occur, while the highest probability is associated to the $\mathrm{AC}$ and $\mathrm{BC}$ pairs. Additionally, for $\mathrm{AB}$ pair, the inclusion of the environment substantially affects the $S_{1}$ while $S_{2}$ remains unchanged. Our results suggest that the inclusion of the immediate adjacent molecules surrounding the pair does not change electronic coupling qualitatively, indicating that the AC and BC pairs still exhibit larger $V$ values than the $\mathrm{AB}$ pair.

Table 2 compares the calculated exciton descriptors for the three tetracene pairs in the gas phase and with the QM/MM 


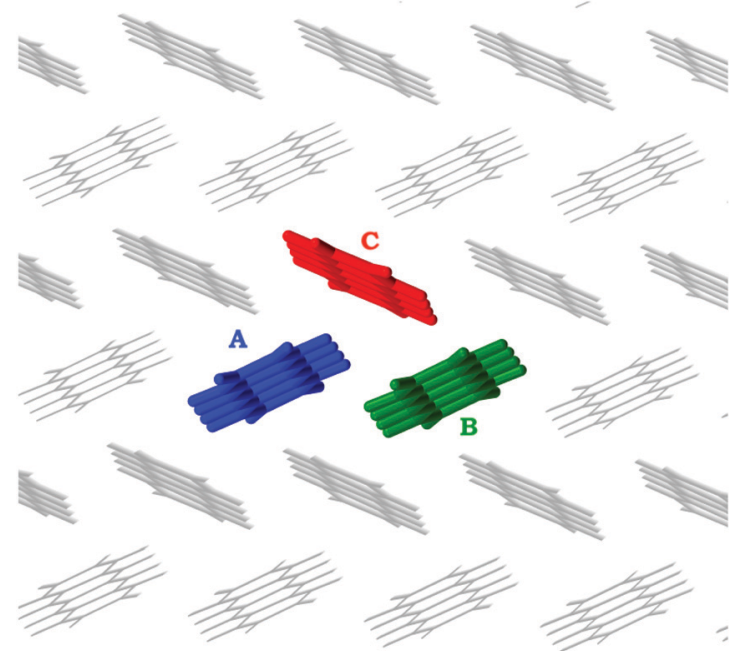

Fig. 5 Crystal structure of tetracene. In the center, the three tetracene molecules included in the QM region are highlighted, and in grey the molecules considered in the MM region.

Table 1 Charged-mediated electronic couplings (in meV) of the photoexcited states with the ${ }^{1} \mathrm{TT}$ state in the three tetracene pairs. Charge transfer states were allowed to mix with both states. S[1] and S[2] represent the diabatic states constructed in the basis of the $\mathrm{S}_{0} \mathrm{~S}_{1}$ and $\mathrm{S}_{1} \mathrm{~S}_{0}$ MEBFs

\begin{tabular}{lrrrrr}
\hline & \multicolumn{2}{c}{ Gas phase } & & \multicolumn{2}{c}{ Embedded cluster } \\
\cline { 2 - 3 } Pair & $V_{\mathrm{S}[1]}$ & $V_{\mathrm{S}[2]}$ & & $V_{\mathrm{S}[1]}$ & $V_{\mathrm{S}[2]}$ \\
\hline $\mathrm{AB}$ & 3.3 & 3.7 & 0.9 & 3.6 \\
$\mathrm{AC}$ & 41.8 & 14.8 & & 54.2 & 17.6 \\
$\mathrm{BC}$ & 23.9 & 11.3 & 40.5 & 13.6 \\
\hline
\end{tabular}

approach. The distance between the centers of mass of the molecules are also shown for each pair. As explained in Section 1.2, the exciton descriptors can be used to assign the character of the excited states of the pairs.

For the $\mathrm{AB}$ pair in the gas phase model, $\mathrm{S}_{1}$ is predicted as the brightest state. The value of $d_{\mathrm{h} \rightarrow \mathrm{e}}$ is zero for all four states, which suggest a LE character. Nevertheless, $d_{\text {exc }}$ reveals that the character of the $S_{1}$ and $S_{2}$ states is different from $S_{3}$ and $S_{4}$. The $\omega_{\mathrm{CT}}$ values give more information about the real character of the excited states. For $S_{1}$ and $S_{2}, \omega_{\mathrm{CT}} \approx 0$ points at a ER character which is further supported by the positive values of $R_{\text {eh }}$ revealing the states as highly correlated bound excitons. In the case of the next two states, $\omega_{\mathrm{CT}} \approx 1$ would suggest a CT character but the negative values of $R_{\mathrm{eh}}$ clarifies that they actually correspond to CR states. Interestingly, we observed that in this pair the excitation energies of the two CR states are higher than the LE ones by $0.40 \mathrm{eV}$. A recent work on pentacene clusters studied the dependency of the relative CT state energies with respect to different functionals and cluster size. The results indicate that low-lying CT states become accessible at larger cluster sizes, and these are predicted by optimallytuned functionals but not by common hybrid ones. The work encourages further studies beyond the dimer model. ${ }^{42}$ The $\mathrm{QM} / \mathrm{MM}$ exciton descriptors for the first two states of the $\mathrm{AB}$ pair $\left(S_{1}\right.$ and $\left.S_{2}\right)$ reveal that they remain ER states as in the gas phase model. Nevertheless, the character of the $S_{3}$ and $S_{4}$ states has changed, and are now better described as CT states. This assignment is beyond doubt as it is based on the combination of the non-zero values of $d_{\mathrm{exc}}=5.537 \AA$ and the low positive values of $R_{\text {eh }}$ of approximately 0.016 . Even though the character changed, the CT states remain higher in energy than the ER ones. In both the gas phase and QM/MM framework, the $S_{1}$ and $\mathrm{S}_{2}$ states turn out to be degenerate $(3.06 \mathrm{eV}$ in gas phase and $3.05 \mathrm{eV}$ in $\mathrm{QM} / \mathrm{MM})$ as well as $\mathrm{S}_{3}$ and $\mathrm{S}_{4}(\approx 3.465 \mathrm{eV}$ in gas phase and $\approx 3.46 \mathrm{eV}$ in $\mathrm{QM} / \mathrm{MM}$ ) which is a consequence of the symmetric parallel relative orientation of the molecules in the $\mathrm{AB}$ pair. Please note that the $\mathrm{AB}$ dimer possesses an inversion center whereas $\mathrm{AC}$ and $\mathrm{BC}$ do not. Our results put in evidence that the inclusion of the environment has a large effect on the exciton descriptors of this pair, and subsequently in the character of the excited states. We speculate that in this disposition SF is not favoured due to the formation of pure ER states without any CT-mixing.

The excited-state character and order in the pairs AC and BC are similar, both in gas phase and $\mathrm{QM} / \mathrm{MM}$ framework. The recognition of the state character is less straightforward than in the $\mathrm{AB}$ pair but, even then, the exciton descriptors facilitate their assignment. In the gas phase, $\mathrm{S}_{1}$ and $\mathrm{S}_{4}$ can be assigned as pure separated CT states since $d_{\mathrm{h} \rightarrow \mathrm{e}}>3.2 \AA$ and $\omega_{\mathrm{CT}}>0.69$. Based on the same descriptors, $S_{2}$ and $S_{3}$ are identified as LE since their $d_{\mathrm{h} \rightarrow \mathrm{e}}$ are less than $1 \AA$ and the $\omega_{\mathrm{CT}}$ values are closer to zero. Nevertheless, the fact that these descriptors are nonzero hints to a non-negligible CT contribution to the character of both LE states. Less obvious indications on the different state character are given in the $d_{\text {exc }}$ where for the CT states the values is higher than $6 \AA$. Unlike the AB pair, the CT and LE states are now closer in energy. Although the inclusion of the environment does not change the character of the four states since two CT and two LE are still identified, the state order is affected by the electrostatic embedding. In this sense that the two lowest singlet states under the QM/MM approach have LE character with small CT contribution while $\mathrm{S}_{3}$ and $\mathrm{S}_{4}$ correspond to the CT states. Despite this new state order, the LE and CT character are observed for these two pairs in contrast with the ER observed in the $\mathrm{AB}$ pair.

The electron-hole correlation plots of the tetracene pairs are shown in Fig. 6. These plots provide an insight beyond the descriptors on where the excitons might be located and from where the CT occurs within the chosen fragmentation scheme. Please note that we consider each molecule as an individual fragment. For the pair $\mathrm{AB}$, it is confirmed that $\mathrm{S}_{1}$ and $\mathrm{S}_{2}$ correspond to ER states whereas $\mathrm{S}_{3}$ and $\mathrm{S}_{4}$ correspond to $\mathrm{CR}$ states. Interestingly, no grey off-diagonal contributions are predicted in the plots of the ER states which indicates no CT contribution. The pattern in the correlation plots change for the $\mathrm{AB}$ pair when the environment is included in the description. The QM/MM correlation plots (second row in Fig. 6) corroborates that the $S_{1}$ and $S_{2}$ states remain with ER character as in the gas phase. Nevertheless, $\mathrm{S}_{3}$ and $\mathrm{S}_{4}$ are now predicted as CT states while in the gas phase they were initially identified as CR states. 
Table 2 Exciton descriptors of the four lowest singlet excited states of the tetracene pairs calculated at the CAM-B3LYP/ANO-S-VDZP level of theory in the gas phase and with a QM/MM approach employing an electrostatic embedding scheme. Excitonic resonance (ER), charge resonance (CR), locally excited (LE) and charge transfer (CT) states are assigned for each of the states. Distance between the centers of mass of the molecules is reported

\begin{tabular}{|c|c|c|c|c|c|c|c|c|c|c|}
\hline Pair & Distance $(\AA)$ & System & State & $\Delta E(\mathrm{eV})$ & $f$ & $\omega_{\mathrm{CT}}$ & $d_{\mathrm{exc}}(\AA)$ & $d_{\mathrm{h} \rightarrow \mathrm{e}}(\AA)$ & $R_{e h}$ & Character \\
\hline \multirow[t]{8}{*}{$\mathrm{AB}$} & \multirow[t]{8}{*}{6.35} & \multirow[t]{4}{*}{ Gas phase } & $\mathrm{S}_{1}$ & 3.06 & 0.406 & 0.01 & 4.689 & 0.000 & 0.458 & ER \\
\hline & & & $\mathrm{S}_{2}$ & 3.06 & 0.000 & 0.02 & 4.731 & 0.000 & 0.459 & ER \\
\hline & & & $\mathrm{S}_{3}$ & 3.46 & 0.002 & 0.97 & 7.353 & 0.000 & -0.374 & CR \\
\hline & & & $\mathrm{S}_{4}$ & 3.47 & 0.000 & 0.98 & 7.377 & 0.000 & -0.385 & CR \\
\hline & & \multirow[t]{4}{*}{$\mathrm{QM} / \mathrm{MM}$} & $\mathrm{S}_{1}$ & 3.05 & 0.402 & 0.01 & 4.684 & 0.011 & 0.457 & ER \\
\hline & & & $\mathrm{S}_{2}$ & 3.05 & 0.001 & 0.02 & 4.727 & 0.004 & 0.458 & ER \\
\hline & & & $\mathrm{S}_{3}$ & 3.45 & 0.001 & 0.97 & 7.366 & 5.537 & 0.017 & CT \\
\hline & & & $\mathrm{S}_{4}$ & 3.47 & 0.000 & 0.98 & 7.368 & 5.537 & 0.015 & CT \\
\hline \multirow[t]{8}{*}{$\mathrm{AC}$} & \multirow[t]{8}{*}{5.20} & \multirow[t]{4}{*}{ Gas phase } & $\mathrm{S}_{1}$ & 2.84 & 0.037 & 0.83 & 6.352 & 3.995 & 0.052 & CT \\
\hline & & & $\mathrm{S}_{2}$ & 2.92 & 0.094 & 0.04 & 4.662 & 0.123 & 0.084 & LE \\
\hline & & & $\mathrm{S}_{3}$ & 3.10 & 0.169 & 0.16 & 5.014 & 0.683 & 0.096 & LE \\
\hline & & & $\mathrm{S}_{4}$ & 3.55 & 0.003 & 0.78 & 6.222 & 3.752 & 0.063 & $\mathrm{CT}$ \\
\hline & & \multirow[t]{4}{*}{$\mathrm{QM} / \mathrm{MM}$} & $\mathrm{S}_{1}$ & 2.90 & 0.085 & 0.06 & 4.734 & 0.249 & 0.105 & LE \\
\hline & & & $\mathrm{S}_{2}$ & 2.97 & 0.123 & 0.44 & 5.621 & 2.069 & 0.084 & LE \\
\hline & & & $\mathrm{S}_{3}$ & 3.16 & 0.085 & 0.54 & 5.820 & 2.591 & 0.068 & CT \\
\hline & & & $\mathrm{S}_{4}$ & 3.35 & 0.008 & 0.92 & 6.512 & 4.439 & 0.044 & $\mathrm{CT}$ \\
\hline \multirow[t]{8}{*}{ BC } & \multirow[t]{8}{*}{5.50} & \multirow[t]{4}{*}{ Gasphase } & $\mathrm{S}_{1}$ & 2.80 & 0.076 & 0.69 & 6.024 & 3.221 & 0.055 & CT \\
\hline & & & $\mathrm{S}_{2}$ & 2.97 & 0.059 & 0.16 & 4.951 & 0.701 & 0.215 & LE \\
\hline & & & $\mathrm{S}_{3}$ & 3.07 & 0.178 & 0.20 & 5.082 & 0.488 & 0.111 & LE \\
\hline & & & $\mathrm{S}_{4}$ & 3.55 & 0.011 & 0.87 & 6.310 & 4.077 & 0.057 & CT \\
\hline & & \multirow[t]{4}{*}{$\mathrm{QM} / \mathrm{MM}$} & $\mathrm{S}_{1}$ & 2.88 & 0.160 & 0.21 & 5.066 & 0.655 & 0.068 & LE \\
\hline & & & $\mathrm{S}_{2}$ & 3.00 & 0.083 & 0.14 & 4.940 & 0.256 & 0.185 & LE \\
\hline & & & $\mathrm{S}_{3}$ & 3.14 & 0.045 & 0.81 & 6.228 & 2.742 & -0.067 & CT \\
\hline & & & $\mathrm{S}_{4}$ & 3.34 & 0.035 & 0.84 & 6.277 & 3.694 & 0.015 & CT \\
\hline
\end{tabular}

These changes highlight how the inclusion of the environment influences the character of the higher excited states as further confirmed the character assignments discussed based on the exciton descriptors reported in Table 2.

The gas phase correlation plots of the AC pair (third row in Fig. 6), further confirm that that $S_{1}$ and $S_{4}$ correspond to CT states. The LE character of $S_{2}$ and $S_{3}$ is also corroborated by the corresponding plots with the excitons located in molecules $\mathrm{C}$ and A, respectively. The QM/MM plots for this pair (fourth row in Fig. 6) show the influence that the environment has on the state order and character. We observe that the $S_{2}$ and $S_{3}$ states in the QM/MM approach resemble linear combinations of the $S_{1}$ and $S_{3}$ states in the gas phase. $S_{1}$ now corresponds to a $L E$ state with the exciton localised in molecule C. $\mathrm{S}_{2}$ (one of the linear combinations) has also LE character with the exciton now localised in molecule A. It is worth to note a pronounced off-diagonal element in the QM/MM plot of $S_{2}$ (second plot in fourth row of Fig. 6) which suggest a higher CT contribution than the similar LE state (third plot in third row of Fig. 6) in the gas phase. $\mathrm{S}_{3}$ and $\mathrm{S}_{4}$ are now the CT states, with $\mathrm{S}_{3}$ (the second of the linear combinations) having a notable LE contribution to its character. For the AC dimer, we have computed the excited state character and their relative ordering under the conductorlike polarizable continuum model ${ }^{79}$ (C-PCM) approach employing three different values of the dielectric constant (3, 5 and 40) as shown in Fig. S1 and Table S1 of the ESI. $\dagger$ The state character and order in the C-PCM analysis is similar to that obtained with the electrostatic embedding in the QM/MM approach.
A similar analysis can be done for the BC pair. In the gas phase plots (fifth row of Fig. 6), $S_{1}$ and $S_{4}$ have CT character whereas $S_{2}$ and $S_{3}$ correspond to the LE states with the excitons localised in molecules $\mathrm{C}$ and $\mathrm{B}$, respectively. When the environment is switched on, the resulting QM/MM plots (last row in Fig. 6) show that the state order and character has changed compared to the corresponding plots in the gas phase. $S_{1}$ and $S_{2}$ correspond to LE states with exciton being localized on molecule $\mathrm{C}$ and $\mathrm{B}$, respectively. $\mathrm{S}_{3}$ and $\mathrm{S}_{4}$ can be easily assigned to CT states based on the correlation plots. The character of the states in the three dimers for both the gas phase and the QM/MM environment is further validated with the corresponding MEBFs coefficients of each state as shown in Tables S2-S7 in the ESI. $\dagger$

The QM/MM results show how the nature of the excited states change with both (i) the orientation of the molecules in the pairs and (ii) when the environment is taken into account. Despite these changes, the underlying mechanism remains similar for both the gas phase and the QM/MM approach. We observed that the LE states are mixed with CT character for the pairs with $V \gg 0$, which highlights that this mixing is responsible for the enhancement of the electronic coupling. Both, the electronic couplings and exciton descriptors, when including the environment, point to a CT-mediated superexchange mechanism, as in our previously reported work. ${ }^{70}$

\section{DPBF}

Panel (a) of Fig. 7 shows the long-range packing of the two known polymorphs of DPBF referred as $\alpha$ and $\beta$ forms. Experimental 


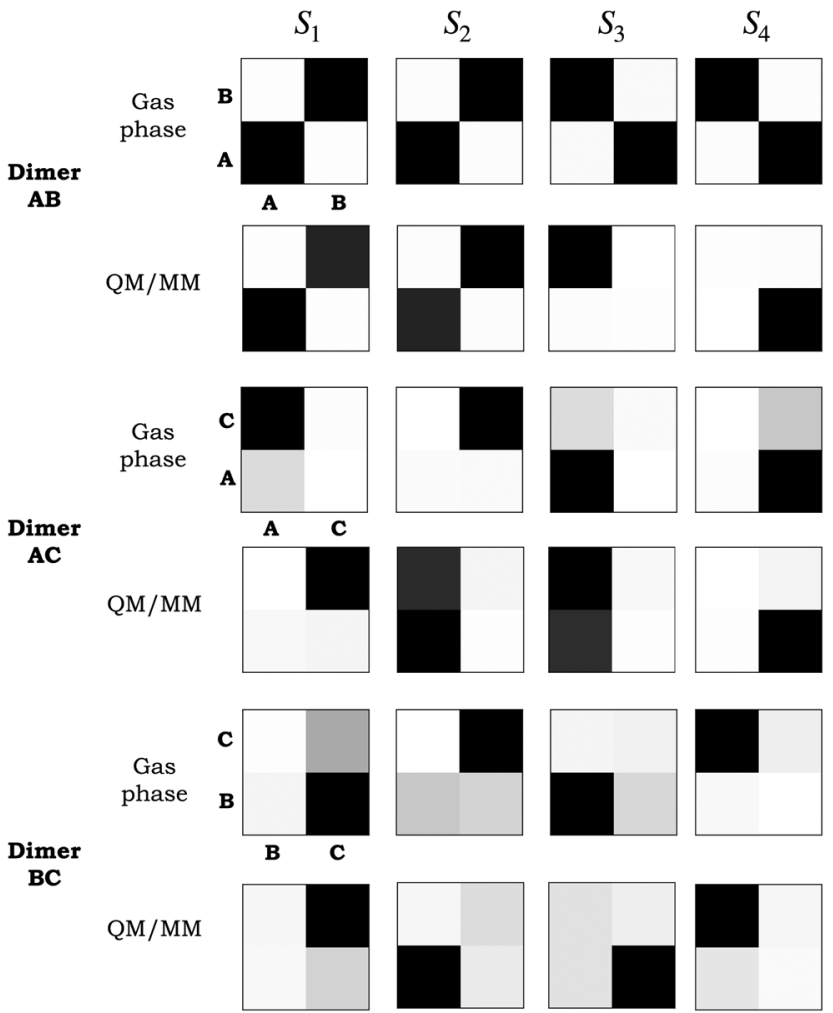

Fig. 6 Electron-hole correlation plots of the three tetracene pair arrangements within the crystal structure in the gas phase and with the QM/MM approach. Pairs were treated at the CAM-B3LYP/ANO-S-VDZP level of theory in the QM region, while the rest of the crystal structure was included in the MM region as electrostatic embedding.

measurements on polycrystalline thin films of both polymorphs revealed that the $\alpha$ form exhibits SF with different yields depending on the temperature, whereas fluorescence predominates in the $\beta$ form quenching the formation of the ${ }^{1}$ TT state. ${ }^{80}$ Both polymorphs have almost an identical crystal structure: they consist of
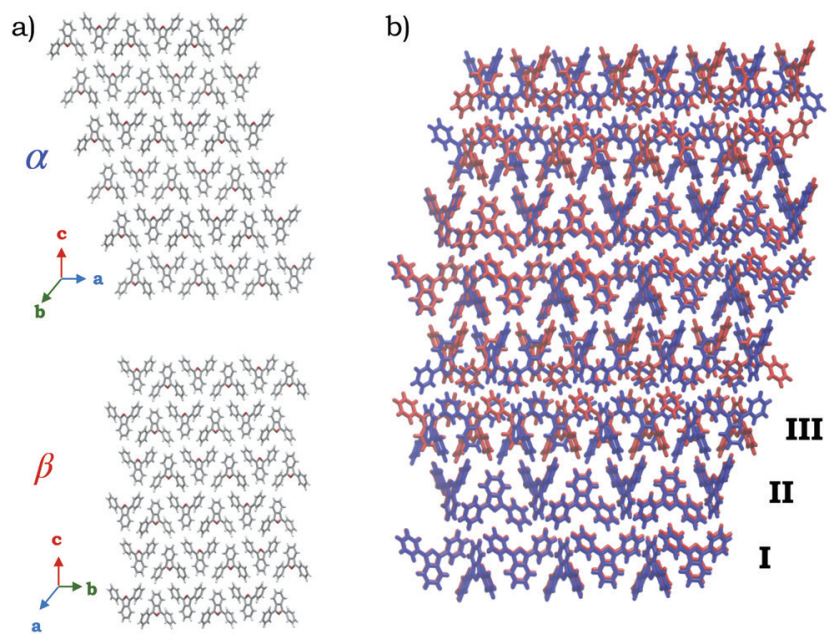

Fig. 7 The two polymorphs of DPBF. (a) The long-range packing of the $\alpha$ and $\beta$ forms are depicted. Vector lengths are indicated in each polymorph. (b) Superposition of a portion of the $\alpha$ (blue) and $\beta$ (red) polymorphs. Nearest-neighbours are almost identical (rows I and II) whereas next-nearest are different (row III). slipped-stacked and herringbone pairs and the possible nearestneighbour pairs ${ }^{35}$ are practically the same in both forms. The only difference between the two forms lies in the next-nearest neighbour interactions. ${ }^{35}$ For clarity, a superposition of a portion of the $\alpha$ and $\beta$ polymorphs is shown in panel (b) of Fig. 7. Here, it is clear that the nearest-neighbour pairs formed between the molecules in rows I and II are similar for both polymorphs. The major difference appears when a next-nearest neighbour molecule (from row III) is considered into the description. This stresses that a gas-phase dimer model would not be enough to explain the difference in SF efficiency between the two, and that the inclusion of the crystal environment is essential. Here, we apply the exciton descriptors to unravel differences in the excited states in both polymorphs where each DPBF molecule is considered as an individual fragment.

We have considered six different QM regions of DPBF (for the gas phase and the $\mathrm{QM} / \mathrm{MM}$ calculations) which were identified within and between the stacks of the polymorphs (see Fig. 8). Two of them correspond to dimers found within the stack and a superposition of these is depicted in panel (a) of Fig. 9. As can be seen, the dimers are almost identical for both polymorphs. One can define another two additional dimer arrangements between the stacks formed by molecules in row I and II. The superposition (panel (b) of Fig. 9) puts in evidence that they are also nearly identical. The other two QM regions consist of trimer arrangements that include one nearest and one next-nearest neighbours between stacks for both polymorphs. It is clear from the superposition, shown in panel (c) of Fig. 9, that the differences between the polymorphs are in the next-nearest neighbour molecules (molecule III).

Table 3 collects the charged-mediated electronic couplings for the dimer and trimers formed for molecules I, II and III in the $\alpha$ and $\beta$ polymorphs. A NOCI calculation on a trimer is not

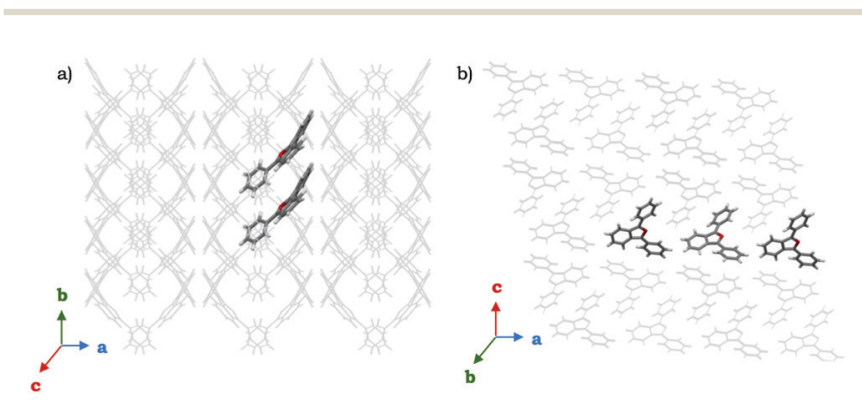

Fig. 8 Depiction of the (a) dimer identified within the stack, and (b) the trimer arrangement formed between stacks in the crystal structure of the $\alpha$ polymorph of DPBF.

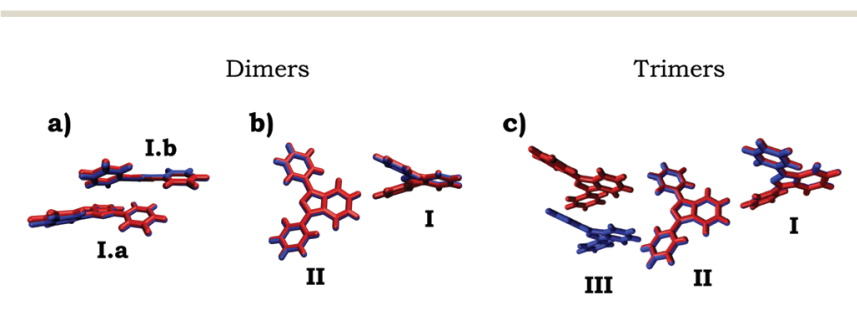

Fig. 9 The QM regions of DPBF treated in this contribution. (a) Dimer arrangements identified within the stack; (b) dimers identified between the stacks and (c) trimer arrangements between different stacks of the $\alpha$ (blue) and $\beta$ (red) polymorphs. 
Table 3 Charged-mediated electronic couplings (in $\mathrm{meV}$ ) of the photoexcited states with the ${ }^{1} T$ states in the dimers and trimers of DPBF. Charge transfer states were allowed to mix with both states. Subscript $X$ in $\Phi_{S[X]}$ indicates for the photoexcited states where the exciton is located, and $X, Y$ in $\Phi_{T T[X, Y]}$ represents in which monomers the triplets are placed

\begin{tabular}{|c|c|c|c|c|c|c|}
\hline Polymorph & & & System & $\Phi_{\mathrm{S}[\mathrm{I}]}$ & $\Phi_{\mathrm{S}[\mathrm{II}]}$ & $\Phi_{\mathrm{S}[\mathrm{III}]}$ \\
\hline \multirow[t]{6}{*}{$\alpha$} & \multirow[t]{2}{*}{ Dimer I-II } & \multirow{2}{*}{$\Phi_{\mathrm{TT}[\mathrm{I}, \mathrm{II}]}$} & Gas phase & 0.7 & 0.6 & - \\
\hline & & & $\mathrm{QM} / \mathrm{MM}$ & 19.7 & 28.3 & - \\
\hline & \multirow[t]{4}{*}{ Trimer } & \multirow[t]{2}{*}{$\Phi_{\mathrm{TT}[\mathrm{I}, \mathrm{II}]}$} & Gas phase & 3.7 & 1.6 & 0 \\
\hline & & & QM/MM & 21.2 & 28.1 & 0 \\
\hline & & \multirow[t]{2}{*}{$\Phi_{\mathrm{TT}[\mathrm{II}, \mathrm{III}]}$} & Gas phase & 0 & 1.9 & 0.7 \\
\hline & & & $\mathrm{QM} / \mathrm{MM}$ & 0 & 28.3 & 37.8 \\
\hline \multirow[t]{6}{*}{$\beta$} & \multirow[t]{2}{*}{ Dimer I-II } & \multirow[t]{2}{*}{$\Phi_{\mathrm{TT}[\mathrm{I}, \mathrm{II}]}$} & Gas phase & 0.7 & 0.7 & - \\
\hline & & & $\mathrm{QM} / \mathrm{MM}$ & 4.1 & 2.9 & - \\
\hline & \multirow[t]{4}{*}{ Trimer } & \multirow[t]{2}{*}{$\Phi_{\mathrm{TT}[1, \mathrm{II}]}$} & Gas phase & 0.7 & 0.4 & 0 \\
\hline & & & $\mathrm{QM} / \mathrm{MM}$ & 8.9 & 2.7 & 0 \\
\hline & & \multirow[t]{2}{*}{$\Phi_{\mathrm{TT}[\mathrm{II}, \mathrm{III}]}$} & Gas phase & 0 & 0.1 & 0.1 \\
\hline & & & QM/MM & 0 & 0.1 & 0.1 \\
\hline
\end{tabular}

yet feasible with the current implementation. For this case, the Hamiltonian and overlap matrix elements were obtained employing the $a b$ initio Frenkel-Davydov exciton model ${ }^{47,81-84}$ (AIFDEM) which is in principle a similar methodology to NOCI. In a previous comparison for dimers in the gas phase, we have established that the couplings calculated with the NOCI and AIFDEM methodologies have a qualitative agreement. ${ }^{16}$ The AIFDEM calculations reveal that the dimers in both polymorphs have similar couplings in the gas phase $(0.7 \mathrm{meV})$, which is not unexpected because of the almost identical conformation of both dimers. Inclusion of environment leads to a larger enhancing in $\alpha$ than in $\beta$. A similar trend is observed for the trimers, where the couplings are enhanced within the $\mathrm{QM} / \mathrm{MM}$ approach in comparison with their gas phase counterparts. Larger values are $(>21.2 \mathrm{meV})$ calculated for $\alpha$ which implies that SF occurs faster than in $\beta$ where the couplings are $<8.9$ meV. A previous study has suggested that besides electronic factors, such as energies and couplings, entropic contributions might play an important role in the solids to explain differences in SF yields. ${ }^{85}$

Table 4 collects the exciton descriptors calculated for the dimer parallel arrangements within the stack (panel (a) in Fig. 9) of the $\alpha$ and $\beta$ forms in the gas phase and the QM/MM description. As expected, the exciton descriptors of the four states calculated in the gas phase are similar for both dimers taken from both polymorphs since they are almost identical. This leads to identical state character and order. Exciton descriptors for $S_{1}$ and $S_{2}$ states suggest in principle a LE character since the $d_{\mathrm{h} \rightarrow \mathrm{e}}$ and $\omega_{\mathrm{CT}}$ are almost zero and the larger $R_{\mathrm{eh}}$ values of approximately 0.47 . Nevertheless, the electron-hole correlation plots in Fig. 10 unravel that they correspond to ER states since the exciton are predicted to be delocalised over the two molecules. $\mathrm{S}_{2}$ is predicted as the bright state. For $\mathrm{S}_{3}$ and $\mathrm{S}_{4}, \omega_{\mathrm{CT}} \approx 1$ to and the negative signs of $R_{\mathrm{eh}}$ point to a CR character which is also confirmed by the corresponding electron-hole correlation plots. Interestingly, the $d_{\mathrm{h} \rightarrow \mathrm{e}}$ values calculated for the CR states $\left(\mathrm{S}_{3}\right.$ and $S_{4}$ ) in the dimer from $\beta$ in the gas phase are lower than those in the dimer from $\alpha$ by $0.2 \AA$. This points to a lesser CT contribution to the character of the states in the $\beta$ polymorph.

The $\mathrm{QM} / \mathrm{MM}$ results for the dimer from the $\beta$ polymorph show that the order of the states and their character are similar to their correspondence in the gas phase with $S_{1}$ and $S_{2}$ identified as ER states and $\mathrm{S}_{3}$ and $\mathrm{S}_{4}$ as $\mathrm{CR}$ ones. These assignments are based on both the exciton descriptors and the electron-hole correlation plots at the bottom of Fig. 10. The similar values on the exciton descriptors and the identical correlation plots suggest that the character of the states in the dimer within the stacks of the $\beta$ form are not affected when the environment is switched on.

In contrast to the results for $\beta$, the character of the states in the dimer from $\alpha$ is highly affected under the presence of the environment within the QM/MM framework. The $S_{1}$ and $S_{2}$ states have now LE character with CT contribution inferred by

Table 4 Exciton descriptors calculated at the CAM-B3LYP/ANO-S-VDZP for the identified DPBF dimers within the stack in the $\alpha$ and $\beta$ polymorphs in the gas phase and with electrostatic embedding (QM/MM approach)

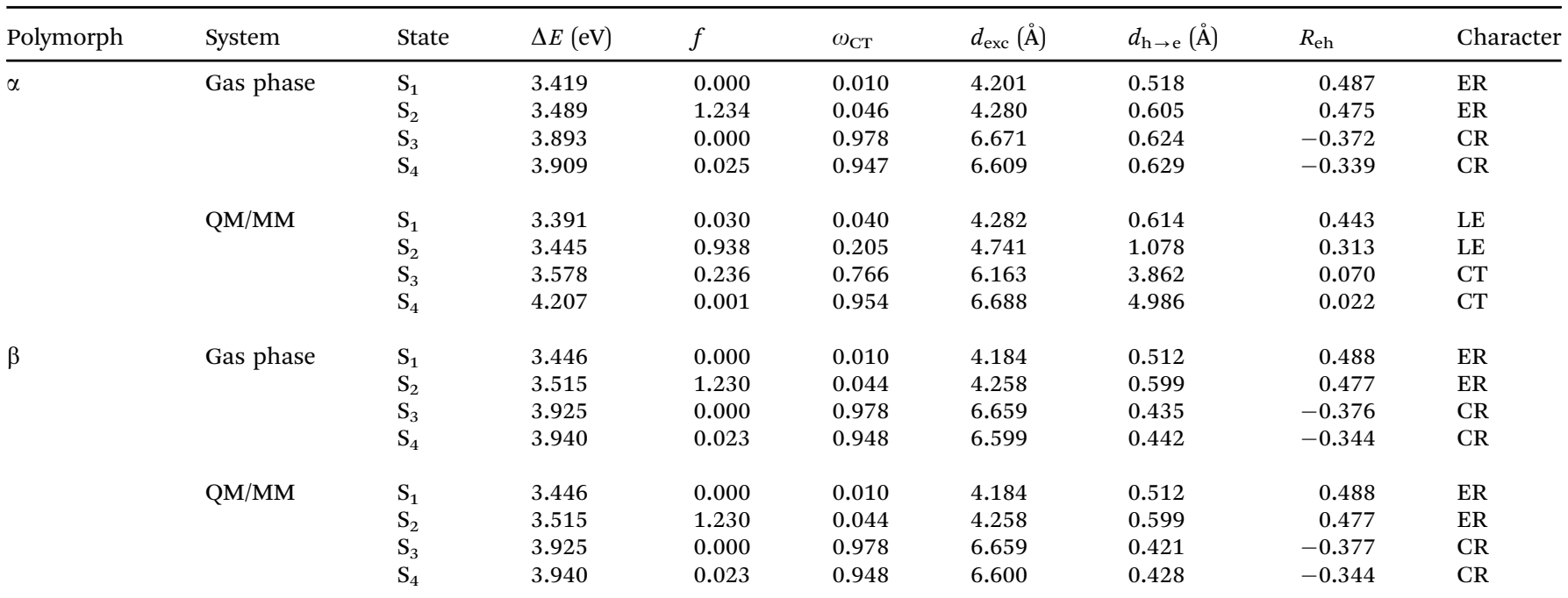




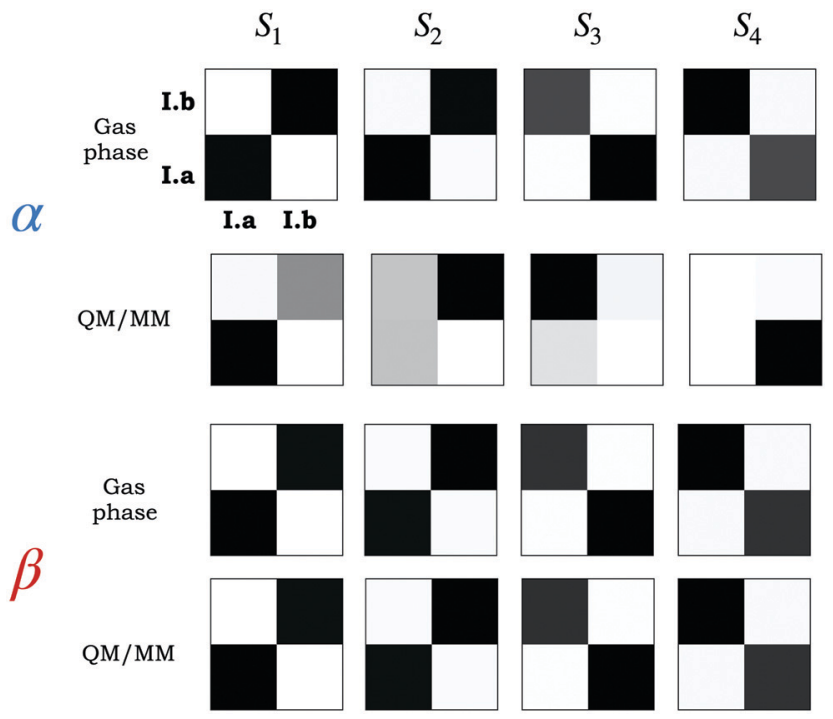

Fig. 10 Electron-hole correlation plots of the DPBF dimers identified within the stacks in the two polymorphs in the gas phase and with the QM/ MM approach.

the gray off-diagonal elements and the non-zero values of the $d_{\mathrm{h} \rightarrow \mathrm{e}}$ and $\omega_{\mathrm{CT}}$ exciton descriptors. This is contrary to the gasphase results where $S_{1}$ and $S_{2}$ are identified as ER states. Additionally, the characters of $S_{3}$ and $S_{4}$ are affected as well; they were identified as CR states in the gas phase, while within the QM/MM approach they correspond to CT states, easily assigned by the large $d_{\mathrm{h} \rightarrow \mathrm{e}}$ of $3.862 \AA$ (for $\mathrm{S}_{3}$ ) and $4.986 \AA$ (for $\mathrm{S}_{4}$ ) and their $\omega_{\mathrm{CT}}$ values close to one (0.766 and 0.954). The $\mathrm{QM} / \mathrm{MM} R_{\mathrm{eh}}$ values of 0.070 and $0.022\left(\mathrm{~S}_{3}\right.$ and $\mathrm{S}_{4}$, respectively) for the CT states suggest that the hole and electron are loosely correlated but bound by Coulombic force. This is again contrary to the gas phase results where the electron and hole are expected to avoid each other $\left(R_{\mathrm{eh}}=-0.372\right.$ and -0.339$)$. The different character of the states in the dimer from $\alpha$ becomes clearer when one looks at the correlation plots in Fig. 10. The $\mathrm{QM} / \mathrm{MM}$ plots confirm that $\mathrm{S}_{1}$ and $\mathrm{S}_{2}$ correspond to LE states (excitons being localized on I.a and I.b, respectively) with CT contributions, and also show the CT character of the $\mathrm{S}_{3}$ and $\mathrm{S}_{4}$ states. This is in contrast with the resonance states predicted in the gas phase plots.

Our results reveal that considering the dimer model in the gas phase is not adequate to explain the experimentally observed difference in SF yields of the polymorphs, and clear differences between the dimers are observed when the environment is switched on.

In sum, our results highlight that character of excited states in the dimer from $\alpha$ is highly affected by the inclusion of the environment compared to the dimer from $\beta$. We observe the presence of LE and CT states in $\alpha$, which exhibits SF, whereas resonance states are observed in $\beta$. Results for the slipped-stack pairs are not enough to explain the difference in SF efficiency. In fact, a previous study has shown that the largest calculated excitonic couplings and SF matrix elements occurs between molecules that are not collocated within the slipped stacks. This conclusion indicates the study should be focused primarily in molecules between different stacks and not within the stack $^{80}$ (see Fig. 8). Despite this conclusion, the QM/MM exciton descriptors put in evidence differences between the polymorphs for the similar dimers when the environment is considered. We observe a localisation of excitons within the slipped stacks in the $\alpha$ form which is in line with experimental evidence of the delocalisation not being great in either polymorph. ${ }^{49}$ We speculate that these local excitons could undergo SF more efficiently with neighboring stack molecules than the ER states formed in the $\beta$ form.

The exciton descriptors for the two dimers formed between stacks (panel (b) in Fig. 9) are presented in Table 5. As expected the state character and order in the gas phase for both dimers from the polymorphs is identical. $S_{1}$ and $S_{2}$ are assigned as LE states while $\mathrm{S}_{4}$ and $\mathrm{S}_{5}$ are CT ones. The character is further

Table 5 Exciton descriptors calculated at the CAM-B3LYP/ANO-S-VDZP for the identified DPBF dimers between stacks in the $\alpha$ and $\beta$ polymorphs in the gas phase and with electrostatic embedding (QM/MM approach)

\begin{tabular}{|c|c|c|c|c|c|c|c|c|c|}
\hline Polymorph & System & State & $\Delta E(\mathrm{eV})$ & $f$ & $\omega_{\mathrm{CT}}$ & $d_{\text {exc }}(\AA)$ & $d_{\mathrm{h} \rightarrow \mathrm{e}}(\AA)$ & $R_{\mathrm{eh}}$ & Character \\
\hline \multirow[t]{8}{*}{$\alpha$} & \multirow[t]{4}{*}{ Gas phase } & $\mathrm{S}_{1}$ & 3.491 & 0.687 & 0.001 & 4.175 & 0.588 & 0.726 & LE \\
\hline & & $\mathrm{S}_{2}$ & 3.496 & 0.717 & 0.000 & 4.174 & 0.584 & 0.726 & $\mathrm{LE}$ \\
\hline & & $\mathrm{S}_{3}$ & 4.536 & 0.000 & 1.000 & 10.840 & 9.875 & -0.003 & CT \\
\hline & & $\mathrm{S}_{4}$ & 4.544 & 0.000 & 0.998 & 10.547 & 9.587 & -0.002 & CT \\
\hline & \multirow[t]{4}{*}{$\mathrm{QM} / \mathrm{MM}$} & $\mathrm{S}_{1}$ & 3.447 & 0.647 & 0.001 & 4.175 & 0.832 & 0.219 & $\mathrm{LE}$ \\
\hline & & $\mathrm{S}_{2}$ & 3.466 & 0.670 & 0.000 & 4.174 & 0.752 & 0.219 & LE \\
\hline & & $\mathrm{S}_{3}$ & 4.399 & 0.000 & 0.999 & 10.384 & 9.423 & -0.002 & $\mathrm{CT}$ \\
\hline & & $\mathrm{S}_{4}$ & 4.643 & 0.000 & 0.999 & 10.978 & 10.031 & -0.003 & $\mathrm{CT}$ \\
\hline \multirow[t]{8}{*}{$\beta$} & \multirow[t]{4}{*}{ Gas phase } & $\mathrm{S}_{1}$ & 3.517 & 0.685 & 0.001 & 4.158 & 0.582 & 0.723 & $\mathrm{LE}$ \\
\hline & & $\mathrm{S}_{2}$ & 3.522 & 0.713 & 0.000 & 4.156 & 0.578 & 0.722 & LE \\
\hline & & $\mathrm{S}_{3}$ & 4.567 & 0.000 & 1.000 & 10.801 & 9.841 & -0.003 & CT \\
\hline & & $\mathrm{S}_{4}$ & 4.574 & 0.000 & 0.998 & 10.519 & 9.565 & -0.003 & $\mathrm{CT}$ \\
\hline & \multirow[t]{4}{*}{$\mathrm{QM} / \mathrm{MM}$} & $\mathrm{S}_{1}$ & 3.545 & 0.734 & 0.001 & 4.175 & 0.410 & 0.529 & $\mathrm{LE}$ \\
\hline & & $\mathrm{S}_{2}$ & 3.554 & 0.764 & 0.000 & 4.184 & 0.365 & 0.528 & LE \\
\hline & & $\mathrm{S}_{3}$ & 3.751 & 0.000 & 0.999 & 10.642 & 9.692 & -0.003 & CT \\
\hline & & $\mathrm{S}_{4}$ & 4.662 & 0.061 & 0.000 & 4.557 & 1.711 & 0.090 & LE \\
\hline
\end{tabular}




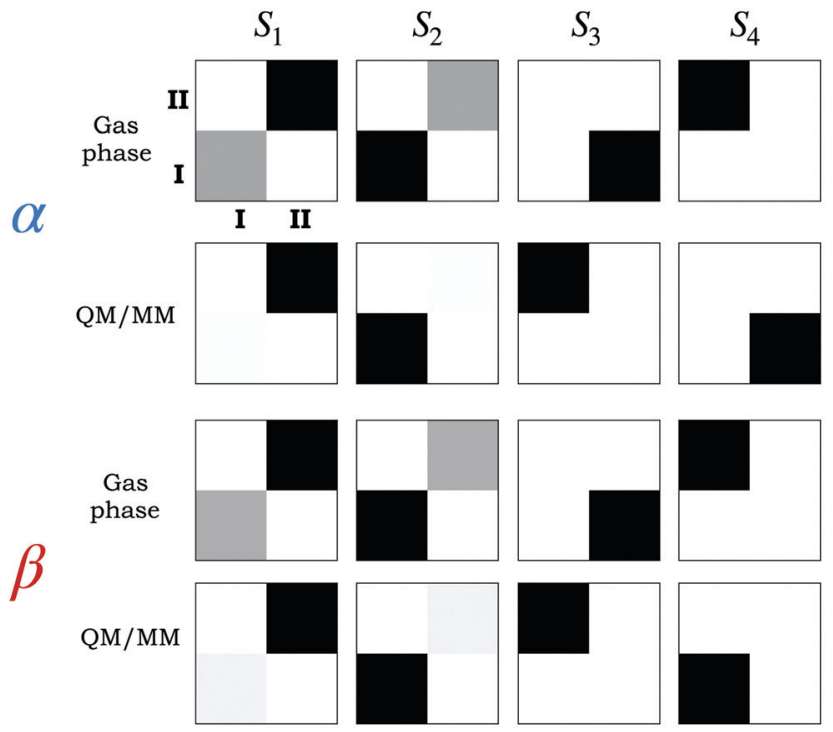

Fig. 11 Electron-hole correlation plots of the DPBF dimers identified between the stacks in the two polymorphs in the gas phase and with the QM/MM approach.

confirmed by the corresponding electron-hole correlation plots depicted in the first and third row of Fig. 11. The nearly identical values of the exciton descriptors for the four states, as well as the similar correlation plots, confirms that the states are practically the same in the gas phase for both dimers. Please note that the the largest difference between this interstack dimers and the previously discussed intrastack dimers is the relative orientation of the two molecules, which reflects already how this affects, at first, the character of the excited states.

Changes are appreciated when the environment is switched on although they are less pronounced. For these two dimers, we observed a localisation of the excitons in the $S_{1}$ and $S_{2}$ states in the QM/MM plots (first and third rows in Fig. 11) whereas the $S_{3}$ $\mathrm{S}_{4}$ remain as CT states but the order has exchanged compared with the gas phase results. Similar QM/MM results for the dimers suggest that inclusion of the environment within the electrostatic embedding scheme is not able to capture totally the effect that the next-nearest molecules has on excited states (in row III) and that inclusion of a explicit third molecule in the QM regions might capture further effects.

Table 6 reports the exciton descriptors calculated for the trimer arrangements. For both trimers in the gas phase, the first three lowest states $\left(S_{1}, S_{2}\right.$ and $\left.S_{3}\right)$ are identified as LE with minor CT contributions. This assignment is done based on the zero values of $\omega_{\mathrm{CT}}$ and $d_{\mathrm{h} \rightarrow \mathrm{e}}<0.6 \AA$. Comparing the descriptors of these three states in the gas phase for both polymorphs, we observe that the $d_{\mathrm{exc}}$ and $d_{\mathrm{h} \rightarrow \mathrm{e}}$ values are almost identical. Nevertheless, the corresponding $R_{\mathrm{eh}}$ values indicate that the LE character of the $S_{1}(0.721$ for $\alpha$ and 0.836 for $\beta$ ) and $S_{2}(0.918$ for $\alpha$ and 0.828 for $\beta$ ) states is different, whereas the descriptors for the $\mathrm{S}_{3}$ are similar for both polymorphs. These differences point to bound excitons but with different degree of correlation and character. The electron-hole correlation plots shine light on these differences. If we compare the gas phase plots (first and third rows in Fig. 12) we can identify them. In the case of $\mathrm{S}_{1}$, the exciton is predicted to be localised in molecule II in $\alpha$ whereas in the $\beta$ it would be

Table 6 Exciton descriptors calculated at the CAM-B3LYP/ANO-S-VDZP for the identified DPBF trimers in the $\alpha$ and $\beta$ polymorphs in the gas phase and with electrostatic embedding (QM/MM approach)

\begin{tabular}{|c|c|c|c|c|c|c|c|c|c|}
\hline Polymorph & System & State & $\Delta E(\mathrm{eV})$ & $f$ & $\omega_{\mathrm{CT}}$ & $d_{\text {exc }}(\AA)$ & $d_{\mathrm{h} \rightarrow \mathrm{e}}(\AA)$ & $R_{\mathrm{eh}}$ & Character \\
\hline \multirow[t]{12}{*}{$\alpha$} & \multirow[t]{6}{*}{ Gasphase } & $\mathrm{S}_{1}$ & 3.489 & 0.778 & 0.000 & 4.176 & 0.588 & 0.721 & LE \\
\hline & & $\mathrm{S}_{2}$ & 3.491 & 0.051 & 0.000 & 4.175 & 0.583 & 0.918 & $\mathrm{LE}$ \\
\hline & & $\mathrm{S}_{3}$ & 3.498 & 1.257 & 0.000 & 4.174 & 0.590 & 0.903 & LE \\
\hline & & $\mathrm{S}_{4}$ & 4.527 & 0.000 & 0.998 & 10.553 & 9.593 & -0.002 & CT \\
\hline & & $\mathrm{S}_{5}$ & 4.530 & 0.000 & 1.000 & 10.852 & 9.888 & -0.003 & CT \\
\hline & & $\mathrm{S}_{6}$ & 4.548 & 0.000 & 0.998 & 10.545 & 9.584 & -0.002 & CT \\
\hline & \multirow[t]{6}{*}{$\mathrm{QM} / \mathrm{MM}$} & $\mathrm{S}_{1}$ & 3.443 & 0.640 & 0.000 & 4.174 & 0.829 & 0.747 & LE \\
\hline & & $\mathrm{S}_{2}$ & 3.447 & 0.446 & 0.000 & 4.173 & 0.836 & 0.803 & LE \\
\hline & & $\mathrm{S}_{3}$ & 3.466 & 0.857 & 0.000 & 4.174 & 0.755 & 0.574 & LE \\
\hline & & $\mathrm{S}_{4}$ & 4.405 & 0.000 & 0.999 & 10.384 & 9.422 & -0.002 & CT \\
\hline & & $\mathrm{S}_{5}$ & 4.497 & 0.000 & 0.998 & 10.360 & 9.394 & -0.002 & CT \\
\hline & & $\mathrm{S}_{6}$ & 4.538 & 0.000 & 1.000 & 11.024 & 10.086 & -0.003 & CT \\
\hline \multirow[t]{12}{*}{$\beta$} & \multirow[t]{6}{*}{ Gasphase } & $\mathrm{S}_{1}$ & 3.515 & 0.369 & 0.000 & 4.159 & 0.583 & 0.836 & LE \\
\hline & & $\mathrm{S}_{2}$ & 3.516 & 0.412 & 0.000 & 4.159 & 0.575 & 0.828 & LE \\
\hline & & $\mathrm{S}_{3}$ & 3.526 & 1.291 & 0.000 & 4.157 & 0.585 & 0.904 & LE \\
\hline & & $\mathrm{S}_{4}$ & 4.559 & 0.000 & 0.998 & 10.521 & 9.567 & -0.003 & CT \\
\hline & & $\mathrm{S}_{5}$ & 4.562 & 0.000 & 1.000 & 10.810 & 9.851 & -0.003 & CT \\
\hline & & $\mathrm{S}_{6}$ & 4.578 & 0.000 & 0.998 & 10.519 & 9.563 & -0.002 & CT \\
\hline & \multirow[t]{6}{*}{$\mathrm{QM} / \mathrm{MM}$} & $\mathrm{S}_{1}$ & 3.529 & 0.719 & 0.000 & 4.169 & 0.529 & 0.332 & LE \\
\hline & & $\mathrm{S}_{2}$ & 3.540 & 0.176 & 0.000 & 4.175 & 0.432 & 0.867 & LE \\
\hline & & $\mathrm{S}_{3}$ & 3.553 & 1.288 & 0.000 & 4.181 & 0.398 & 0.859 & LE \\
\hline & & $\mathrm{S}_{4}$ & 3.776 & 0.000 & 1.000 & 19.807 & 19.305 & 0.000 & CT \\
\hline & & $\mathrm{S}_{5}$ & 3.925 & 0.000 & 0.999 & 10.604 & 9.647 & -0.003 & CT \\
\hline & & $\mathrm{S}_{6}$ & 3.950 & 0.000 & 0.999 & 10.584 & 9.636 & -0.003 & CT \\
\hline
\end{tabular}




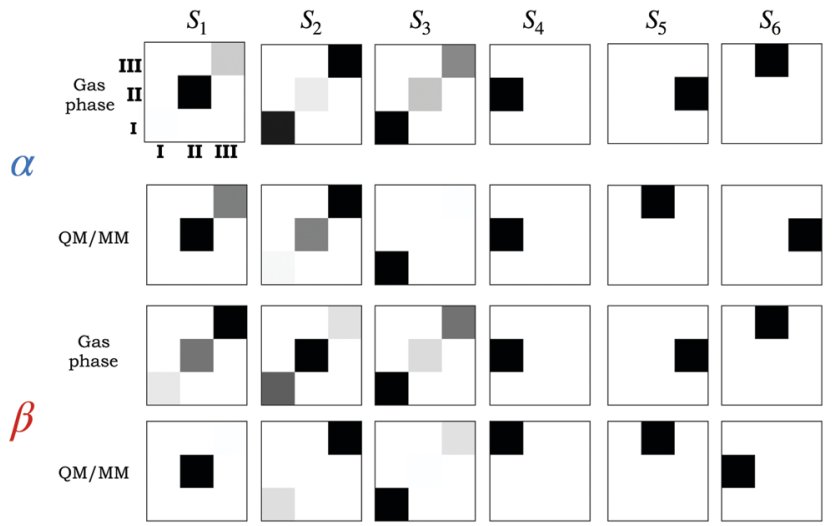

Fig. 12 Electron-hole correlation plots of the DPBF trimers identified in the two polymorphs in the gas phase and with the QM/MM approach.

localised predominantly in molecule III (refer to Fig. 9). The differences in the $S_{2}$ state are even more pronounced since the exciton in the trimer from $\alpha$ is predicted to be delocalised mainly between molecules I and III whereas in $\beta$ it would correspond to a delocalised exciton predominantly in molecules I and II. The almost identical plots for $\mathrm{S}_{3}$ (predicted as the brightest state since it has the larger $f$ value) confirm that this state has similar character for both trimers in the gas phase, an exciton delocalised over the three molecules with the major contribution coming from molecule I.

The gas phase exciton descriptors for the $\mathrm{S}_{4}, \mathrm{~S}_{5}$ and $\mathrm{S}_{6}$ states lead to a CT character in both polymorphs. The assignment can be safely done by the $\omega_{\mathrm{CT}} \approx 1$ and the $d_{\mathrm{exc}}>9 \AA$. Interestingly, direct comparison of the exciton descriptors for each of these three states between both polymorphs suggest a similar character in both trimers since the values of the descriptors are almost identical. The electron-hole correlation plots (Fig. 12) indicate from where to where the CT occurs. The gas phase plots for $\alpha$ (first row) show that in the $\mathrm{S}_{4}$ state there is CT from molecule I to II. In the case of $\mathrm{S}_{5}$, the CT occurs from molecule III to II and in $\mathrm{S}_{6}$ the transfer goes from molecule II to III. The gas phase correlation plots of $\mathrm{S}_{4}, \mathrm{~S}_{5}$ and $\mathrm{S}_{6}$ of the trimer from $\beta$ (third row) are almost identical to the ones in $\alpha$ which confirms that the character is the same for these states in both trimers of the polymorphs.

For both polymorphs, the QM/MM descriptors for the trimers show that the $S_{1}, S_{2}$ and $S_{3}$ states have LE character, as in the gas phase results, since $\omega_{\mathrm{CT}}=0.000$ and the close-to-zero $d_{\mathrm{h} \rightarrow \mathrm{e}}$ values. It is worth to point out that the QM/MM $d_{\mathrm{h} \rightarrow \mathrm{e}}$ values of the states in the $\beta$ form are lower than those in the $\alpha$ form, e.g. for $\mathrm{S}_{1}, R_{\mathrm{eh}}=$ 0.829 in $\alpha$ and 0.529 in $\beta$; for $\mathrm{S}_{2}, R_{\mathrm{eh}}=0.836$ in $\alpha$ and 0.432 in $\beta$; and for $\mathrm{S}_{3}, R_{\mathrm{eh}}=0.755$ in $\alpha$ and 0.398 in $\beta$. These changes are also reflected in the correlation plots in Fig. 12. For the $S_{1}$ state, the correlation plot indicate that the exciton in the $\alpha$ form is delocalised between molecules II and III (first plot from the second row) whereas in the $\beta$ form the exciton is predominantly localised on molecule II (first plot in the fourth row). As in $\mathrm{S}_{1}$, the exciton formed in $\mathrm{S}_{2}$ is also delocalised. In the trimer extracted from the $\beta$ polymorph, there is a localised exciton on molecule III with a minor delocalisation onto molecule I (second plot in fourth row in Fig. 12). This is contrast with $S_{2}$ calculated for the $\alpha$ polymorph, where the delocalisation is more pronounced particularly across molecules II and III (second plot in second row in Fig. 12). For $S_{3}$, the plots for both polymorphs are almost identical, suggesting a similar character with the exciton predominantly localised on molecule I with minor contributions from molecule III.

$\mathrm{S}_{4}, \mathrm{~S}_{5}$ and $\mathrm{S}_{6}$ states are straightforwardly assigned as CT states from the QM/MM exciton descriptors in Table 6 in both polymorphs. The character follows immediately from $\omega_{\mathrm{CT}} \approx 1$ and $d_{\text {exc }}>9 \AA$. It is important to note that the corresponding $R_{\text {eh }}$ values of these states range from 0.000 to -0.003 for both polymorphs. These values suggest that the electron and hole generated after the CT are either not correlated or avoiding each other in an almost negligible degree. However, there is a major difference in the $\mathrm{S}_{4}$ state for both polymorphs. $d_{\mathrm{h} \rightarrow \mathrm{e}}$ is $9.422 \AA$ and $19.305 \AA$ for the $\alpha$ and $\beta$ forms, respectively. This points to a larger separation in space of the charges in this particular state of the trimer from $\beta$. The $d_{\mathrm{h} \rightarrow \mathrm{e}}$ values for $\mathrm{S}_{5}$ are similar for both polymorphs (9.394 and $9.647 \AA$ ) and slightly different for $\mathrm{S}_{6}(10.086$ and $9.636 \AA$ ).

The electron-hole correlation plots offer an insight on where the exciton might be located. For the $\mathrm{S}_{4}$ state, the plots reveal that the CT in $\alpha$ occurs from molecule I to II (dark off-diagonal element in the fourth graph in the second row) whereas in the $\beta$ form the CT occurs from molecule I to III (dark off-diagonal element in the fourth graph in the fourth row). This explains the difference in the distance between the charges in both polymorphs. The plots of the $S_{5}$ state are identical for $\alpha$ and $\beta$ with the CT occurring from molecule II to III. For $S_{6}$, the CT occurs from molecule III to II in the case of $\alpha$ and from molecule I to II in the $\beta$ form.

We observe that, for both polymorphs, the QM/MM plots show a localisation of the excitons in the $S_{1}, S_{2}$ and $S_{3}$ states in comparison with the gas phase, particularly in the $\beta$ form. Experimental evidence suggest that in films of the $\beta$ form loss of energy occurs due to presence of low energy states, such as excimers or traps. ${ }^{80}$ Our QM/MM results hint to two main differences: (1) the exciton formed in $S_{3}$ (the brightest state) of the $\beta$ form has a higher correlation $\left(R_{\mathrm{eh}}=0.859\right)$ than the one in the brightest state $\left(\mathrm{S}_{3}\right)$ in the $\alpha$ polymorph $\left(R_{\mathrm{eh}}=0.574\right)$. This high correlation suggests that the excitons formed are more tightly bound in the $\beta$ form, and this might hinder the conversion to the ${ }^{1}$ TT state; and (2) the possible formation of CT states leads to larger separated electron and hole $(\approx 19 \AA)$ in the case of the $\beta$ form which could function as a trap since the generated charges are not correlated $\left(R_{\mathrm{eh}} \approx 0\right)$ and might move freely through the crystal.

Comparison of the gas phase correlation plots of the dimers (first and third rows in Fig. 11) and trimer (first and third rows in Fig. 12) arrangements reveals that the changes are not particularly significant. The gas phase plots of the trimers (first and third rows of Fig. 12) show that adding the third molecule in both trimers give slightly different state character for two of the LE states, i.e. $\mathrm{S}_{1}$ and $\mathrm{S}_{2}$. The $\mathrm{S}_{3}, \mathrm{~S}_{4}, \mathrm{~S}_{5}$ and $\mathrm{S} 6$ states seem to 
be similar for both trimers. Differences are already appreciated when we consider the $\mathrm{QM} / \mathrm{MM}$ results. In the case of the trimer extracted from the $\alpha$ polymorph, inclusion of the environment results in excitons predominantly localised in one of the molecules, i.e. molecule II in $\mathrm{S}_{1}$, molecule III in $\mathrm{S}_{2}$ and molecule I in $S_{3}$ (second row of Fig. 12). As can be seen, the localisation in the $\mathrm{QM} / \mathrm{MM}$ plots is less pronounced in $\alpha$ than in $\beta$ for the above mentioned states. The environment seems to have a larger effect for the LE states in the trimer from the $\beta$ polymorph than in the trimer from $\alpha$.

In overall, the QM/MM exciton descriptors are able to spot differences between the two polymorphs (for the dimers and trimer arrangements considered) which highlights the importance of going beyond the gas-phase dimer model for certain chromophores and the potential of the excited state analysis to explore and unravel differences for other polymorphic systems. As for tetracene, the AIFDEM eigenvectors (Tables S8-S19 in the $\mathrm{ESI} \dagger$ ) corroborate the character of the identified states employing TD-DFT in the gas phase and within the QM/MM approach in the dimers and trimers of DPBF.

\section{Conclusions}

To summarize, we have explored the influence of the crystal packing on the electronic couplings and the character of excited state of different molecular arrangements of tetracene and DPBF, two chromophores known to exhibit singlet fission.

Three tetracene pairs were identified within the crystal structure exhibiting different electronic coupling values. These couplings are enhanced when the environment is considered for two of these pairs ( $\mathrm{AC}$ and $\mathrm{BC}$ ), while in the parallel $\mathrm{AB}$ disposition this is not observed. The results indicate that, even though the numerical value changes, the inclusion of surrounding adjacent molecules do not change the electronic couplings qualitatively with $\mathrm{AC}$ and $\mathrm{BC}$ exhibiting larger values than $\mathrm{AB}$. Gas phase exciton descriptors for the tetracene pairs revealed that the excited character of the dimers is affected, in the first instance, by the mutual disposition of the molecules. On top of this, inclusion of the environment affects the excited state character and order of the dimers particularly on $\mathrm{AB}$. Within the $\mathrm{QM} / \mathrm{MM}$ framework, we observed LE and CT states in the pairs with non-zero electronic coupling (AC and BC), and ER and CT states in AB. Despite these differences, the electronic couplings and exciton descriptors show that the LE states mix with CT ones which points to a CT-mediated superexchange mechanism in both the gas phase and the QM/MM approach.

We illustrated how the excited state analysis can be used to unravel differences between two different polymorphs, namely $\alpha$ and $\beta$, which exhibit different SF yields with $\alpha$ showing the higher SF efficiency. First, we identified dimer arrangements within the stacks of the polymorphs. These dimer arrangements are almost identical in both the $\alpha$ and $\beta$ forms. This leads to a similar excited state character and order in the gas phase. Differences in the excited states of the dimers are solely spotted when the crystal environment is taken into consideration.
We observe ER and CR states in the $\beta$ while in $\alpha$ LE and CT states are expected. We speculate that these local excitons in $\alpha$ might go under SF easier than the resonance states formed in the $\beta$ polymorph.

We also studied the excited states in dimers and trimers formed between molecules in different stacks in the crystal structures of the polymorphs. The results for the trimers stress the effect that the relative orientation of the molecules has, at first, on the excited state character. We identified that, for the trimer arrangements in the $\alpha$ and $\beta$ forms, the differences in the excited state character are more pronounced with the inclusion of the crystal packing within the QM/MM framework. Based on our QM/MM exciton descriptors, we speculate that the exciton formed in the brightest state of the $\beta$ form is stronger correlated than the one formed in the brightest state in the trimer of $\alpha$. We hypothesize that the formed excitons are more tightly bound in $\beta$ than in $\alpha$. This stronger interaction in the $\beta$ polymorph could hinder the conversion of the photoexcited chromophore to the ${ }^{1} \mathrm{TT}$ state explaining its lower SF efficiency. These observations emphasize that the inclusion of the environment is crucial to understand some of the experimental observations where the dimer model might not be adequate or enough.

\section{Conflicts of interest}

There are no conflicts to declare.

\section{Acknowledgements}

This research used resources of the Oak Ridge Leadership Computing Facility located at Oak Ridge National Laboratory, which was supported by the Office of Science of the Department of Energy under Contract No. DEAC05-00OR22725. This work is part of Innovational Research Incentives Scheme Vidi 2017 with project number 016.Vidi.189.044, which is (partly) financed by the Dutch Research Council (NWO). Financial support was also provided by the Spanish Administration (Project CTQ2017-83566-P) and the Generalitat de Catalunya (Project 2017-SGR629).

\section{Notes and references}

1 C. D. Thomas, A. Cameron, R. E. Green, M. Bakkenes, L. J. Beaumont, Y. C. Collingham, B. F. N. Erasmus, M. F. de Siqueira, A. Grainger, L. Hannah, L. Hughes, B. Huntley, A. S. van Jaarsveld, G. F. Midgley, L. Miles, M. A. Ortega-Huerta, A. Townsend Peterson, O. L. Phillips and S. E. Williams, Nature, 2004, 427, 145-148.

2 C. Li, F. Wang and C. Yu, Energy Environ. Sci., 2011, 4, 100-113.

3 J. Barber and P. D. Tran, J. R. Soc., Interface, 2013, 10, 20120984.

4 J.-L. Bredas, J. E. Norton, J. Cornil and V. Coropceanu, Acc. Chem. Res., 2009, 42, 1691-1699.

5 M. B. Smith and J. Michl, Chem. Rev., 2010, 110, 6891-6936.

6 M. B. Smith and J. Michl, Annu. Rev. Phys. Chem., 2013, 64, 361-386. 
7 J. Lee, P. Jadhav, P. D. Reusswig, S. R. Yost, N. J. Thompson, D. N. Congreve, E. Hontz, T. van Voorhis and M. A. Baldo, Acc. Chem. Res., 2013, 46, 1300-1311.

8 D. Casanova, Chem. Rev., 2018, 118, 7164-7207.

9 M. C. Hanna and A. J. Nozik, J. Appl. Phys., 2006, 100, 074510.

10 M. J. Y. Tayebjee, A. A. Gray-Weale and T. W. Schmidt, J. Phys. Chem. Lett., 2012, 3, 2749-2754.

11 B. Ehrler, M. W. B. Wilson, A. Rao, R. H. Friend and N. C. Greenham, Nano Lett., 2012, 12, 1053-1057.

12 P. D. Reusswig, D. N. Congreve, N. J. Thompson and M. A. Baldo, Appl. Phys. Lett., 2012, 101, 113304.

13 C. Dover and F. Gallaher, et al., Nat. Chem., 2018, 10, 305-310.

14 N. Korovina, C. H. Chang and J. Johnson, Nat. Chem., 2020, 12, 391-398.

15 I. Paci, J. C. Johnson, X. Chen, G. Rana, D. Popovia, D. E. David, A. J. Nozik, M. A. Ratner and J. Michl, J. Am. Chem. Soc., 2006, 128, 16546-16553.

16 L. E. A. Suarez, R. K. Kathir, E. Siagri, R. W. A. Havenith and S. Faraji, Adv. Quantum Chem., 2019, 79, 263-287.

17 O. El Bakouri, J. R. Smith and H. Ottosson, J. Am. Chem. Soc., 2020, 142, 5602-5617.

18 T. Zeng, N. Ananth and R. Hoffmann, J. Am. Chem. Soc., 2014, 136, 12638-12647.

19 C. Hetzer, D. M. Guldi and R. R. Tykwinski, Chem. - Eur. J., 2018, 24, 8245-8257.

20 D. Padula, O. H. Omar, T. Nematiaram and A. Troisi, Energy Environ. Sci., 2019, 12, 2412-2416.

21 K. J. Fallon, P. Budden, E. Salvadori, A. M. Ganose, C. N. Savory, L. Eyre, S. Dowland, Q. Ai, S. Goodlett, C. Risko, D. O. Scanlon, C. W. M. Kay, A. Rao, R. H. Friend, A. J. Musser and H. Bronstein, J. Am. Chem. Soc., 2019, 141, 13867-13876.

22 Z. Havlas and J. Michl, Isr. J. Chem., 2016, 56, 96-106.

23 E. A. Buchanan, Z. Havlas and J. Michl, Adv. Quantum Chem., 2017, 75, 175-227.

24 A. Zaykov, P. Felkel, E. A. Buchanan, M. Jovanovic, R. W. A. Havenith, R. K. Kathir, R. Broer, Z. Havlas and J. Michl, J. Am. Chem. Soc., 2019, 141, 17729-17743.

25 J. L. Ryerson, A. Zaykov, L. E. Aguilar Suarez, R. W. A. Havenith, B. R. Stepp, P. I. Dron, J. Kaleta, A. Akdag, S. J. Teat, T. F. Magnera, J. R. Miller, Z. Havlas, R. Broer, S. Faraji, J. Michl and J. C. Johnson, J. Chem. Phys., 2019, 151, 184903.

26 H. Yamagata, J. Norton, E. Hontz, Y. Olivier, D. Beljonne, J. L. Brédas, R. J. Silbey and F. C. Spano, J. Chem. Phys., 2011, 134, 204703.

27 T. C. Berkelbach, M. S. Hybertsen and D. R. Reichman, J. Chem. Phys., 2013, 138, 114103.

28 W.-L. Chan, T. C. Berkelbach, M. R. Provorse, N. R. Monahan, J. R. Tritsch, M. S. Hybertsen, D. R. Reichman, J. Gao and X.-Y. Zhu, Acc. Chem. Res., 2013, 46, 1321-1329.

29 S. Yost, J. Lee and M. Wilson, et al., Nat. Chem., 2014, 6, 492-497.

30 N. Monahan and X.-Y. Zhu, Annu. Rev. Phys., 2015, 66, 601-618.
31 T. Zeng, R. Hoffmann and N. Ananth, J. Am. Chem. Soc., 2014, 136, 5755-5764.

32 A. B. Kolomeisky, X. Feng and A. I. Krylov, J. Phys. Chem. C, 2014, 118, 19608-19617.

33 L. Wang, Y. Olivier, O. V. Prezhdo and D. Beljonne, J. Phys. Chem. Lett., 2014, 5, 3345-3353.

34 M. H. Farag and A. I. Krylov, J. Phys. Chem. C, 2018, 122, 25753-25763.

35 E. A. Buchanan and J. Michl, Photochem. Photobiol. Sci., 2019, 18, 2112-2124.

36 T. C. Berkelbach, M. S. Hybertsen and D. R. Reichman, J. Chem. Phys., 2014, 141, 074705.

37 P. E. Teichen and J. D. Eaves, J. Chem. Phys., 2015, 143, 044118.

38 M. Nakano, T. Nagami, T. Tonami, K. Okada, S. Ito, R. Kishi, Y. Kitagawa and T. Kubo, J. Comput. Chem., 2019, 40, 89-104.

39 M. Wibowo, M. Persico and G. Granucci, Phys. Chem. Chem. Phys., 2019, 21, 692-701.

40 H. Miyamoto and M. Nakano, ChemPhotoChem, 2020, 4, 5249-5263.

41 P. M. Zimmerman, F. Bell, D. Casanova and M. HeadGordon, J. Am. Chem. Soc., 2011, 133, 19944-19952.

42 B. Alam, A. F. Morrison and J. M. Herbert, J. Phys. Chem. C, 2020, 124, 24653-24666.

43 N. Geacintov, M. Pope and F. Vogel, Phys. Rev. Lett., 1969, 22, 593-596.

44 R. P. Groff, P. Avakian and R. E. Merrifield, Phys. Rev. B: Solid State, 1970, 1, 815-817.

45 J. J. Burdett, A. M. Müller, D. Gosztola and C. J. Bardeen, J. Chem. Phys., 2010, 133, 144506.

46 M. Pope, J. Burgos and J. Giachino, J. Chem. Phys., 1965, 43, 3367.

47 A. F. Morrison and J. M. Herbert, J. Phys. Chem. Lett., 2017, 8, 1442-1448.

48 J. Johnson, A. J. Nozik and J. Michl, J. Am. Chem. Soc., 2010, 132, 16302-16303.

49 J. N. Schrauben, J. L. Ryerson, J. Michl and J. C. Johnson, J. Am. Chem. Soc., 2014, 136, 7363-7373.

50 J. Johnson and J. Michl, Top. Curr. Chem., 2017, 375, 1-29.

51 A. Tokmakoff, Fermi's Golden Rule, University of chicago technical report, 2007.

52 Z.-Q. You and C.-P. Hsu, J. Chem. Phys., 2010, 133, 074105.

53 J. C. Johnson, A. J. Nozik and J. Michl, Acc. Chem. Res., 2013, 46, 1290-1299.

54 C. H. Yang and C. P. Hsu, J. Phys. Chem. Lett., 2015, 6, 1925-1929.

55 T. P. Straatsma, R. Broer, S. Faraji and R. W. A. Havenith, Annu. Rep. Comput. Chem., 2018, 14, 77-91.

56 T. P. Straatsma, R. Broer, S. Faraji, R. W. A. Havenith, L. E. A. Suarez, R. K. Kathir, M. Wibowo and C. de Graaf, J. Chem. Phys., 2020, 152, 064111.

57 R. W. A. Havenith, H. D. de Gier and R. Broer, Mol. Phys., 2012, 110, 2445-2454.

58 Z.-Q. You and C.-P. Hsu, Int. J. Quantum Chem., 2014, 114, 102-115.

59 P. Huang and E. A. Carter, Annu. Rev. Phys. Chem., 2008, 59, 261-290. 
60 A. Domingo, A. Rodríguez-Fortea, M. Swart, C. de Graaf and R. Broer, Phys. Rev. B: Condens. Matter Mater. Phys., 2012, 85, 155143.

61 S. A. Bäppler, F. Plasser, M. Wormit and A. Dreuw, Phys. Rev. A: At., Mol., Opt. Phys., 2014, 90, 052521.

62 F. Plasser, M. Wormit and A. Dreuw, J. Chem. Phys., 2014, 141, 024106.

63 F. Plasser, S. A. Bappler, M. Wormit and A. Dreuw, J. Chem. Phys., 2014, 141, 024107.

64 S. A. Mewes, F. Plasser, A. I. Krylov and A. Dreuw, J. Chem. Theory Comput., 2018, 14, 710-725.

65 F. Plasser and H. Lischka, J. Chem. Theory Comput., 2012, 8, 2777-2789.

66 F. Plasser, B. Thomitzni, S. A. Bäppler, J. Wenzel, D. R. Rehn, M. Wormit and A. Dreuw, J. Comput. Chem., 2015, 36, 1609-1620.

67 S. A. Mewes, F. Plasser and A. Dreuw, J. Chem. Phys., 2015, 143, 171101.

68 S. A. Mewes, J.-M. Mewes, A. Dreuw and F. Plasser, Phys. Chem. Chem. Phys., 2016, 18, 2548-2563.

69 S. A. Mewes and A. Dreuw, Phys. Chem. Chem. Phys., 2019, 21, 2843-2856.

70 L. E. A. Suarez, M. F. S. J. Menger and S. Faraji, Mol. Phys., 2020, 118, e1769870.

71 H. M. Senn and W. Thiel, Angew. Chem., Int. Ed., 2009, 48, 1198-1229.

72 I. F. Galván, M. Vacher, A. Alavi, C. Angeli, F. Aquilante, J. Autschbach, J. J. Bao, S. I. Bokarev, N. A. Bogdanov, R. K. Carlson, L. F. Chibotaru, J. Creutzberg, N. Dattani, M. G. Delcey, S. S. Dong, A. Dreuw, L. Freitag, L. M. Frutos, L. Gagliardi, F. Gendron, A. Giussani, L. González, G. Grell, M. Guo, C. E. Hoyer, M. Johansson, S. Keller, S. Knecht, G. Kovačevi, E. Källman, G. Li Manni, M. Lundberg, Y. Ma, S. Mai, J. P. Malhado, P. Malmqvist, P. Marquetand, S. A. Mewes, J. Norell, M. Olivucci, M. Oppel, Q. M. Phung, K. Pierloot, F. Plasser, M. Reiher, A. M. Sand, I. Schapiro, P. Sharma, C. J. Stein, L. K. Sørensen, D. G. Truhlar, M. Ugandi, L. Ungur, A. Valentini, S. Vancoillie, V. Veryazov, O. Weser, T. A. Wesołowski, P.-O. Widmark, S. Wouters, A. Zech, J. P. Zobel and R. Lindh, J. Chem. Theory Comput., 2019, 15, 5925-5964.

73 F. Aquilante, J. Autschbach, A. Baiardi, S. Battaglia, V. A. Borin, L. F. Chibotaru, I. Conti, L. De Vico, M. Delcey, I. F. Galván, N. Ferré, L. Freitag, M. Garavelli, X. Gong, S. Knecht, E. D. Larsson, R. Lindh, M. Lundberg, P. Malmqvist, A. Nenov, J. Norell, M. Odelius, M. Olivucci, T. B. Pedersen, L. Pedraza-González, Q. M. Phung, K. Pierloot, M. Reiher, I. Schapiro, J. Segarra-Martí, F. Segatta, L. Seijo, S. Sen, D.-C. Sergentu, C. J. Stein, L. Ungur, M. Vacher, A. Valentini and V. Veryazov, J. Chem. Phys., 2020, 152, 214117.

74 R. K. Kathir, C. de Graaf, R. Broer and R. W. A. Havenith, J. Chem. Theory Comput., 2020, 16, 2941-2951.

75 Y. Shao, Z. Gan, E. Epifanovsky, A. T. Gilbert, M. Wormit, J. Kussmann, A. W. Lange, A. Behn, J. Deng, X. Feng, D. Ghosh, M. Goldey, P. R. Horn, L. D. Jacobson,
I. Kaliman, R. Z. Khaliullin, T. Kuś, A. Landau, J. Liu, E. I. Proynov, Y. M. Rhee, R. M. Richard, M. A. Rohrdanz, R. P. Steele, E. J. Sundstrom, H. Lee Woodcock III, P. M. Zimmerman, D. Zuev, B. Albrecht, E. Alguire, B. Austin, G. J. O. Beran, Y. A. Bernard, E. Berquist, K. Brandhorst, K. B. Bravaya, S. T. Brown, D. Casanova, C.-M. Chang, Y. Chen, S. H. Chien, K. D. Closser, D. L. Crittenden, M. Diedenhofen, R. A. D. Jr., H. Do, A. D. Dutoi, R. G. Edgar, S. Fatehi, L. Fusti-Molnar, A. Ghysels, A. GolubevaZadorozhnaya, J. Gomes, M. W. Hanson-Heine, P. H. Harbach, A. W. Hauser, E. G. Hohenstein, Z. C. Holden, T.-C. Jagau, H. Ji, B. Kaduk, K. Khistyaev, J. Kim, J. Kim, R. A. King, P. Klunzinger, D. Kosenkov, T. Kowalczyk, C. M. Krauter, K. U. Lao, A. D. Laurent, K. V. Lawler, S. V. Levchenko, C. Y. Lin, F. Liu, E. Livshits, R. C. Lochan, A. Luenser, P. Manohar, S. F. Manzer, S.-P. Mao, N. Mardirossian, A. V. Marenich, S. A. Maurer, N. J. Mayhall, E. Neuscamman, C. M. Oana, R. Olivares-Amaya, D. P. O’Neill, J. A. Parkhill, T. M. Perrine, R. Peverati, A. Prociuk, D. R. Rehn, E. Rosta, N. J. Russ, S. M. Sharada, S. Sharma, D. W. Small, A. Sodt, T. Stein, D. Stück, Y.-C. Su, A. J. Thom, T. Tsuchimochi, V. Vanovschi, L. Vogt, O. Vydrov, T. Wang, M. A. Watson, J. Wenzel, A. White, C. F. Williams, J. Yang, S. Yeganeh, S. R. Yost, Z.-Q. You, I. Y. Zhang, X. Zhang, Y. Zhao, B. R. Brooks, G. K. Chan, D. M. Chipman, C. J. Cramer, W. A. Goddard III, M. S. Gordon, W. J. Hehre, A. Klamt, H. F. Schaefer III, M. W. Schmidt, C. D. Sherrill, D. G. Truhlar, A. Warshel, X. Xu, A. Aspuru-Guzik, R. Baer, A. T. Bell, N. A. Besley, J.-D. Chai, A. Dreuw, B. D. Dunietz, T. R. Furlani, S. R. Gwaltney, C.-P. Hsu, Y. Jung, J. Kong, D. S. Lambrecht, W. Liang, C. Ochsenfeld, V. A. Rassolov, L. V. Slipchenko, J. E. Subotnik, T. V. Voorhis, J. M. Herbert, A. I. Krylov, P. M. Gill and M. Head-Gordon, Mol. Phys., 2015, 113, 184-215.

76 A. V. Marenich, S. V. Jerome, C. J. Cramer and D. G. Truhlar, J. Chem. Theory Comput., 2012, 8, 527-541.

77 F. Plasser, J. Chem. Phys., 2020, 152, 084108.

78 F. Plasser, TheoDORE: a package for theoretical density, orbital relaxation and exciton analysis, http://theodore-qc. sourceforge.net.

79 S. Miertus, E. Scrocco and J. Tomasi, Chem. Phys., 1981, 55, 117-129.

80 J. L. Ryerson, J. N. Schrauben, A. J. Ferguson, S. C. Sahoo, P. Naumov, Z. Havlas, J. Michl, A. J. Nozik and J. C. Johnson, J. Phys. Chem. C, 2014, 118, 12121-12132.

81 A. F. Morrison, Z.-Q. You and J. M. Herbert, J. Chem. Theory Comput., 2014, 10, 5366-5376.

82 A. F. Morrison and J. M. Herbert, J. Phys. Chem. Lett., 2015, 6, 4390-4396.

83 J. M. Herbert, X. Zhang, A. F. Morrison and J. Liu, Acc. Chem. Res., 2016, 49, 931-941.

84 A. F. Morrison and J. M. Herbert, J. Chem. Phys., 2017, 146, 224110.

85 A. B. Kolomeisky, X. Feng and A. I. Krylov, J. Phys. Chem. C, 2014, 118, 5188-5195. 